\title{
The Role of Calcium/Calmodulin-Activated Calcineurin in Rapid and Slow Endocytosis at Central Synapses
}

\author{
Tao Sun, ${ }^{1 \star}$ Xin-Sheng Wu, ${ }^{1 \star}$ Jianhua Xu, ${ }^{1}$ Benjamin D. McNeil, ${ }^{1}$ Zhiping P. Pang, ${ }^{2}$ Wanjun Yang, ${ }^{3}$ Li Bai, ${ }^{1}$ Syed Qadri, ${ }^{1}$ \\ Jeffery D. Molkentin, ${ }^{4}$ David T. Yue, ${ }^{3}$ and Ling-Gang Wu ${ }^{1}$ \\ ${ }^{1}$ National Institute of Neurological Disorders and Stroke, Bethesda, Maryland 20892, 2Department of Molecular and Cellular Physiology, Stanford \\ University, Palo Alto, California 94304-5543, ${ }^{3}$ Department of Biomedical Engineering, Johns Hopkins University School of Medicine, Baltimore, Maryland \\ 21205, and ${ }^{4}$ Department of Pediatrics, University of Cincinnati, Cincinnati Children's Hospital Medical Center, Howard Hughes Medical Institute, \\ Cincinnati, Ohio 45229
}

\begin{abstract}
Although the calcium/calmodulin-activated phosphatase calcineurin may dephosphorylate many endocytic proteins, it is not considered a key molecule in mediating the major forms of endocytosis at synapses-slow, clathrin-dependent and the rapid, clathrin-independent endocytosis. Here we studied the role of calcineurin in endocytosis by reducing calcium influx, inhibiting calmodulin with pharmacological blockers and knockdown of calmodulin, and by inhibiting calcineurin with pharmacological blockers and knock-out of calcineurin. These manipulations significantly inhibited both rapid and slow endocytosis at the large calyx-type synapse in 7- to 10-d-old rats and mice, and slow, clathrin-dependent endocytosis at the conventional cultured hippocampal synapse of rats and mice. These results suggest that calcium influx during nerve firing activates calcium/calmodulin-dependent calcineurin, which controls the speed of both rapid and slow endocytosis at synapses by dephosphorylating endocytic proteins. The calcium/calmodulin/calcineurin signaling pathway may underlie regulation of endocytosis by nerve activity and calcium as reported at many synapses over the last several decades.
\end{abstract}

\section{Introduction}

The calcium/calmodulin-dependent phosphatase calcineurin, found widely in the nervous system (Rusnak and Mertz, 2000), may dephosphorylate many endocytosis proteins, such as dynamin, synaptojanin, the adaptor protein AP180, and phosphatidylinositol phosphate kinase type I $\gamma$ (Clayton et al., 2007). This raises the possibility that calcineurin may mediate the calciumdependent regulation of endocytosis (Cousin and Robinson, 2001), as observed at many synapses (Royle and Lagnado, 2003; $\mathrm{Wu}, 2004$ ). Based on measurements of the FM dye release in the synaptosome preparation, an early study implicated the involvement of calcineurin in endocytosis during extremely intense stimulation, depolarization for hundreds of seconds (Marks and McMahon, 1998). Consistent with this implication, calcineurin is considered to be involved only in bulk endocytosis during very intense stimuli, but not in slow, clathrin-dependent endocytosis during milder stimuli at cerebellar synapses (Evans and Cousin, 2007; Clayton and Cousin, 2009; Clayton et al., 2009). Slow endocytosis at a calyx-type nerve terminal is triggered by $>10 \mu \mathrm{M}$ calcium (Hosoi et al., 2009; X. S. Wu et al., 2009), which is much

\footnotetext{
Received March 23, 2010; revised July 15, 2010; accepted July 18, 2010.

This work was supported by the National Institute of Neurological Disorders and Stroke Intramural Research Program. We thank Drs. Jonathan G. Seidman (Harvard Medical School, Boston, MA) and Jennifer L. Gooch (Emory University School of Medicine, Atlanta, GA) for providing us with calcineurin $\mathrm{A}_{\alpha}^{+/-}$mice. We thank Dr. Gero Miesenböck (University of Oxford, $0 x f$ ord, UK) for providing us with the synaptopHluorin plasmid.

*T.S. and X.-S.W. contributed equally.

Correspondence should be addressed to Ling-Gang Wu, National Institute of Neurological Disorders and Stroke, 35 Convent Drive, Building 35, Room 2B-1012, Bethesda, MD 20892. E-mail: wul@ninds.nih.gov.

DOI:10.1523/JNEUROSCI.1481-10.2010

Copyright $\odot 2010$ the authors $\quad 0270-6474 / 10 / 3011838-10 \$ 15.00 / 0$
}

higher than the affinity of calcineurin to calcium $(\sim 1-1.5 \mu \mathrm{M})$. This result also argues against the involvement of calcineurin in slow endocytosis during milder stimuli (Hosoi et al., 2009). Rapid endocytosis, which is presumably clathrin-independent (Artalejo et al., 1995; Jockusch et al., 2005), is another form of endocytosis often observed at synapses (L. G. Wu et al., 2007). Likely because of its fast speed, calcineurin-mediated dephosphorylation is not considered to be involved in this process. In summary, while calcineurin may dephosphorylate endocytic proteins, there has been no molecular and biophysical evidence showing the involvement of calcineurin in rapid and slow endocytosis, two major forms of endocytosis observed in near physiological stimuli at synapses (Royle and Lagnado, 2003; L. G. Wu et al., 2007).

Recent studies at giant calyx-type synapses suggest that calcium influx triggers and regulates rapid and slow endocytosis (Hosoi et al., 2009; X. S. Wu et al., 2009). The calcium binding protein calmodulin was implied as the calcium receptor, because its blockers significantly inhibited rapid and slow endocytosis (X. S. Wu et al., 2009). However, three main issues had remained unresolved. First, pharmacological blockers may not be specific to calmodulin. Direct molecular biological evidence supporting calmodulin as the calcium sensor for endocytosis is missing, likely because calmodulin is encoded by three dispersed genes in vertebrates, making it difficult to use genetic approaches. Second, if calcium/calmodulin initiates rapid and slow endocytosis, its downstream target is unclear. Although calcineurin has been discussed as a downstream target for a long time, evidence supporting its role in rapid and slow endocytosis is missing. Third, it is unclear whether the findings obtained at giant synapses apply to the majority of synapses, the small conventional synapses. We addressed 


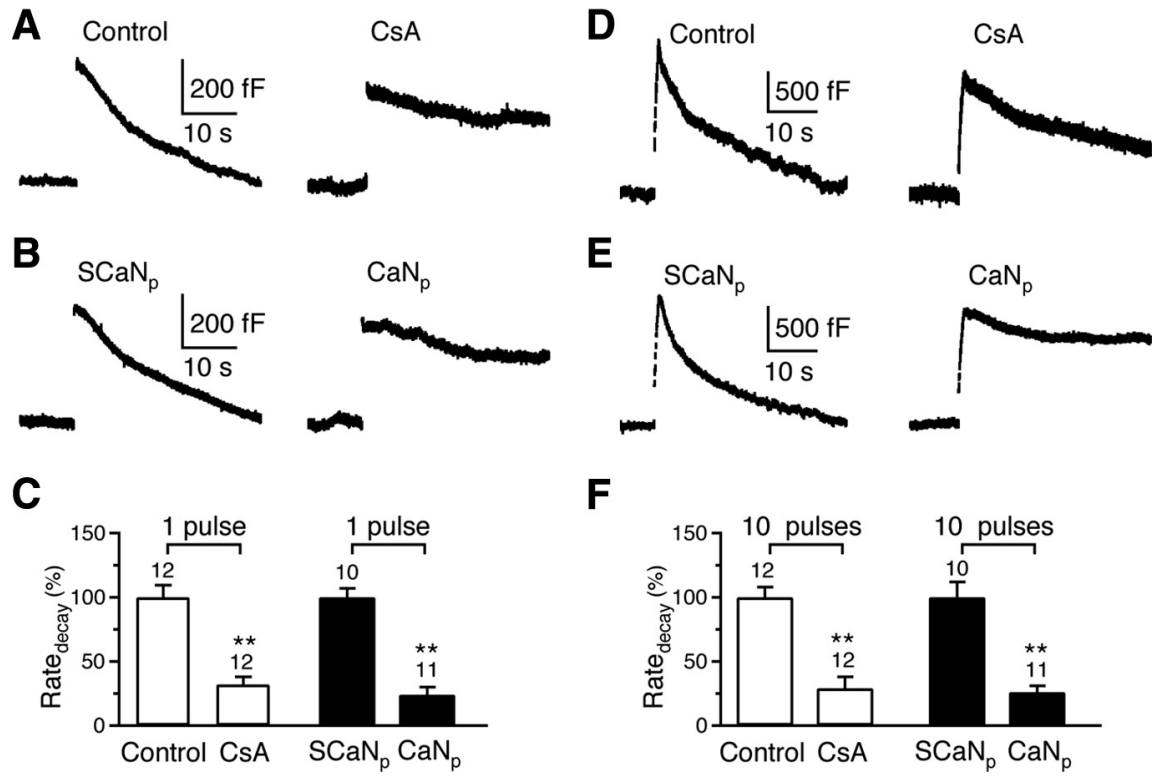

Figure 1. Calcineurin blockers inhibit rapid and slow endocytosis at the calyx. $A$, Two sampled capacitance $\left(C_{m}\right)$ traces showing endocytosis induced by a $20 \mathrm{~ms}$ depolarization with a pipette containing a control solution (with $0.1 \%$ DMSO) or $20 \mu \mathrm{M}$ CsA (with $0.1 \%$ DMSO). Traces in Figures $1-3$ are mostly individual traces and occasionally an average of $2-3$ traces. Data were obtained from rat calyces in Figures 1 and 2 . B. Two sampled $C_{\mathrm{m}}$ traces showing endocytosis induced by a $20 \mathrm{~ms}$ depolarization with a pipette containing scrambled $\mathrm{CaN}_{457-482}\left(\mathrm{SCaN}_{\mathrm{p}}, 150 \mu \mathrm{m}\right.$, serves as control) or $\mathrm{CaN}_{457-482}\left(\mathrm{CaN}_{\mathrm{p}}, 150 \mu \mathrm{m}\right)$. C, Comparison of the Rate ${ }_{\text {decay }}$ after a $20 \mathrm{~ms}$ depolarization in the absence (control) and the presence of $\mathrm{CSA}(20 \mu \mathrm{m})$, and in the presence of $\mathrm{SCaN}_{\mathrm{p}}(150 \mu \mathrm{M})$ or $\mathrm{CaN}_{\mathrm{p}}(150 \mu \mathrm{m})$. The number of calyces tested are labeled (applies to $F$ ). ${ }^{* *} p<0.01$ (applies to Figs. 1-3). The data for CSA and control group (open bars) were normalized to the mean value of the control group, whereas the data for $\mathrm{CaN}_{\mathrm{p}}$ and $\mathrm{SCaN}_{\mathrm{p}}$ group (solid bars) were normalized to the mean value of the $\mathrm{SCaN}_{\mathrm{p}}$ group (applies to Figs. 1-3). Note that this panel aims at showing the inhibitory effect of CsA and $\mathrm{CaN}_{\mathrm{p}}$ compared with their corresponding control. Because of the method of normalizing the data described above, a comparison between the control and the $\mathrm{SCaN}_{\mathrm{p}}$ group is not meaningful (applies to Fig. $1 \mathrm{Fand}$ Fig. 2C). Data are expressed as mean \pm SE (applies to all figures). $\boldsymbol{D}-\boldsymbol{F}$, Similar to $\boldsymbol{A}-\boldsymbol{C}$, respectively, except that the stimulus was 10 pulses of $20 \mathrm{~ms}$ depolarization at $10 \mathrm{~Hz}$, which induced a Rate decay $_{\text {with }}>80 \%$ caused by rapid endocytosis in control.

these three issues by combining quantitative measurements of endocytosis, pharmacological tools, and genetic approaches at both giant calyx-type and small cultured hippocampal synapses. We found that block of the calcium/calmodulin/calcineurin signaling pathway significantly inhibited both rapid and slow endocytosis, which calls for modification of the current endocytosis model to include calcineurin as a key player.

\section{Materials and Methods}

Slice preparation, capacitance recordings, and solutions. Parasagittal brainstem slices (200 $\mu \mathrm{m}$ thick) containing the medial nucleus of the trapezoid body were prepared from 7- to 10-d-old male or female Wistar rats or mice using a vibratome (X. S. Wu et al., 2009). Whole-cell capacitance measurements were made with the EPC-9 amplifier together with the software lock-in amplifier (PULSE, HEKA) that implements LindauNeher's technique (Sun and Wu, 2001; Sun et al., 2004). The frequency of the sinusoidal stimulus was $1000 \mathrm{~Hz}$ and the peak-to-peak voltage of the sine wave was $\leq 60 \mathrm{mV}$. We pharmacologically isolated presynaptic $\mathrm{Ca}^{2+}$ currents with a bath solution $\left(\sim 22-24^{\circ} \mathrm{C}\right)$ containing the following (in $\mathrm{mm}$ ): $105 \mathrm{NaCl}, 20$ TEA-Cl, $2.5 \mathrm{KCl}, 1 \mathrm{MgCl}_{2}, 2 \mathrm{CaCl}_{2}, 25 \mathrm{NaHCO}_{3}, 1.25$ $\mathrm{NaH}_{2} \mathrm{PO}_{4}, 25$ glucose, 0.4 ascorbic acid, 3 myo-inositol, 2 sodium pyruvate, 0.001 tetrodotoxin (TTX), 0.1 3,4-diaminopyridine, $\mathrm{pH} 7.4$ when bubbled with $95 \% \mathrm{O}_{2}$ and $5 \% \mathrm{CO}_{2}$. The presynaptic pipette contained the following (in mM): $125 \mathrm{Cs}$-gluconate, $20 \mathrm{CsCl}, 4 \mathrm{MgATP}, 10 \mathrm{Na}_{2}$-phosphocreatine, 0.3 GTP, 10 HEPES, 0.05 BAPTA, pH 7.2, adjusted with CsOH. Since DMSO $(0.1 \%)$ was used to dissolve CsA (Sigma) in the pipette solution, the control solution for this drug also contained 0.1\% DMSO (Fig. $1 A, D$ ). $\mathrm{CaN}_{457-482}$ and scrambled $\mathrm{CaN}_{457-482}$ were purchased from Calbiochem and GenScript USA Inc, respectively.

Hippocampal cultures and fluorescence imaging. Hippocampal cultures, stimulation and fluorescence imaging were similar to those described previ- ously (Sankaranarayanan and Ryan, 2000). Hippocampal CA1-CA3 regions from postnatal day 0 (P0)-P2 Sprague Dawley rats (if not mentioned) or P0 mice were dissected, dissociated, and plated on Matrigel-coated glass coverslips (BD Biosciences). Cells were maintained at $37^{\circ} \mathrm{C}$ in a $5 \% \mathrm{CO}_{2}$ humidified incubator with a culture media consisting of MEM (Invitrogen), 0.5\% glucose, $0.1 \mathrm{~g} / \mathrm{L}$ bovine transferrin (Calbiochem), $0.3 \mathrm{~g} / \mathrm{L}$ glutamine, $10 \%$ fetal bovine serum (Invitrogen), 2\% B-27 (Invitrogen), and 3 $\mu \mathrm{M}$ cytosine $\beta$-D-arabinofuranoside (Sigma). Six to $8 \mathrm{~d}$ after plating, calcium-phosphatemediated gene transfer was used to transfect cultures with synaptopHluorin $(\mathrm{SpH}$, kindly provided by Dr. G. Miesenböck, University of Oxford, Oxford, UK), calmodulin shRNA plasmid, or calmodulin shRNA-resistant plasmid. After transfection, cultures were maintained at $37^{\circ} \mathrm{C}$ in a $5 \% \quad \mathrm{CO}_{2}$ humidified incubator for another $6-8 \mathrm{~d}$ before use. Unless otherwise indicated, all chemicals were obtained from Sigma.

Coverslips were mounted in a stimulation chamber (RC-21BRFS chamber, Warner Instruments) $6-8 \mathrm{~d}$ after transfection. The action potential was evoked by passing a $1 \mathrm{~ms}$ current pulse of $20 \mathrm{~mA}$ via platinum electrodes in the chamber. The bath solution $\left(\sim 22-24^{\circ} \mathrm{C}\right)$ contained the following (in $\mathrm{mm}$ ): $119 \mathrm{NaCl}, 2.5$ $\mathrm{KCl}, 2 \mathrm{CaCl}_{2}, 2 \mathrm{MgCl}_{2}, 25$ HEPES (buffered to $\mathrm{pH}$ 7.4), 30 glucose, 0.01 6-cyano-7nitroquinoxaline-2, 3-dione (CNQX) and 0.05 d-1-2-amino-5-phosphonovaleric acid (AP-5). When lowering the $\mathrm{CaCl}_{2}$ concentration, $\mathrm{MgCl}_{2}$ was increased to keep the divalent ion concentration constant.

$\mathrm{SpH}$ images were acquired at $1 \mathrm{~Hz}$ using the Zeiss LSM 510 META confocal microscope with a $40 \times, 1.3$ numerical aperture oil-immersion objective. Images were analyzed using Zeiss LSM510 software. All functionally visible varicosities were selected for analysis by testing their responsiveness to stimulation. The fluorescence intensity within a region of at least $1.5 \mu \mathrm{m} \times 1.5 \mu \mathrm{m}$ were averaged together for each bouton, which avoided fluorescence decay caused by faster diffusive processes (Granseth et al., 2006). Each group of data was obtained from at least 3 different batches of cultures.

Calmodulin knockdown, immunostaining, and Western blot. Calmodulin shRNA and calmodulin shRNA-resistant plasmids are described recently (Pang et al., 2010). We made only one modification, i.e., the GFP was cutoff from these plasmids to avoid the fluorescence conflict with cotransfected SpH. Both plasmids include two RNA-polymerase III promoters (human H1 and human U6) in tandem and the Ubiquitin C promoter downstream of U6 promoter. For the calmodulin shRNA plasmid, a shorthairpin sequence targeting a common sequence found in the calmodulin 1 and calmodulin 2 mRNAs (CTGACTGAAGAGCAGATTGC; full shRNA sequence: TCGACCCCTGACTGAAGAGCAGATTGCTTCAAGAGAGCAATCTGCTCTTCAGTCAGTTTTTGGAAAT) was inserted into the downstream of the $\mathrm{H} 1$ promoter. A second short-hairpin sequence targeting the calmodulin 3 mRNA (sequence: CGCGCCCACGGAGCTGCAGGACATGATTATTCAAGAGATAATCATGTCCTGCAGCTCCGTTTTTTGGAAA) was inserted into the downstream of the U6 promoter.

The calmodulin shRNA-resistant plasmid includes not only the two short-hairpin sequences described above to knockdown calmodulin, but also a mutant calmodulin sequence to rescue calmodulin expression. The BamHI-EcoRI sites downstream of ubiquitin C promoter are for the insertion of rescue calmodulin cDNA. The targeted sequences in the rescue calmodulin cDNA were mutated to TTAACGGAAGAACAAATCGC and CAGAACTTCAAGATATGATCA to create a maximum difference between 
the shRNAs and rescue cDNA without changing the calmodulin protein sequence.

For immunostaining, neurons were fixed with $4 \%$ paraformaldehyde, permeabilized with $0.2 \%$ Triton X-100, and subsequently incubated with the primary and secondary antibodies. The antisera were diluted in PBS with $2 \%$ bovine serum albumin and incubated with cells overnight at $4^{\circ} \mathrm{C}$. After several rinses in PBS, cells were incubated with fluorescenceconjugated donkey anti-rabbit IgG (1:100) and rhodamine-conjugated donkey anti-mouse or donkey anti-goat IgG (1:100, Jackson ImmunoResearch Laboratories) for $30 \mathrm{~min}$ at $37^{\circ} \mathrm{C}$. The following antibodies were used for immunocytochemistry: polyclonal rabbit anti-GFP (1:1000, Invitrogen), and monoclonal mouse anti-calmodulin (1:500, Santa Cruz Biotechnology Inc.). Calmodulin expression level was measured at cell bodies and compared with the fluorescence intensity in un-transfected (SpH-negative) neurons. Although calmodulin was also found in neuronal branches, the immunostaining signal was weak and difficult to quantify. Thus, we did not quantify calmodulin level in neuronal branches.

For Western blot of PC12 cells, cells were washed three times with ice-cold PBS. Cell lysates were prepared in the modified RIPA buffer including protease inhibitors. Equal protein amounts were analyzed by SDS-PAGE and immunoblotting using antibodies against calmodulin (1: 1000, Santa Cruz Biotechnology Inc.) and actin (used as an internal control, 1: 10,000, Millipore Bioscience Research Reagents).

For brain tissue Western blot, dissociated hippocampal CA1-CA3 regions of 9-d-old mice were homogenized in the ice-cold, modified RIPA buffer, which included protease inhibitors. The homogenates were centrifuged at $13,000 \mathrm{rpm}$ at $4^{\circ} \mathrm{C}$ for $20 \mathrm{~min}$. The supernatants were loaded to SDS-PAGE for immunoblotting using antibodies against calcineurin $\mathrm{A}_{\alpha}$ subunit (1:200), calcineurin $A_{\beta}$ subunit (1:1000, Santa Cruz Biotechnology Inc.), and actin (1:10,000).

Calcineurin knock-out. Calcineurin $\mathrm{A}_{\alpha}^{+/-}$and $\mathrm{A}_{\beta}^{+/-}$mice were provided by Dr. J. L. Gooch (Emory University School of Medicine, Atlanta, GA) (Zhang et al., 1996) and J. D. Molkentin (Bueno et al., 2002), respectively. Calcineurin $\mathrm{A}_{\alpha}^{-1-}$ and $\mathrm{A}_{\beta}^{-1-}$ mice were obtained by heterozygous breeding using standard mouse husbandry procedures. Mouse genotypes were determined by PCR with primers described previously (Gooch et al., 2004).

Data analysis. The statistical test was $t$ test. Means are presented as \pm

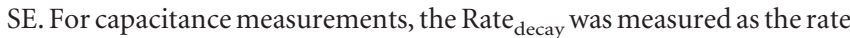
of decay in the first 2-10 s after stimulation. When endocytosis was inhibited, the Rate ${ }_{\text {decay }}$ was measured as the mean decay rate within $10-30 \mathrm{~s}$ after stimulation, because the capacitance decay was approximately linear within this time window. For $\mathrm{SpH}$ signal, the Rate decay was measured as the decay rate in the first $4-10 \mathrm{~s}$ after stimulation. When endocytosis was inhibited, the Rate decay $_{\text {was }}$ measured from the first $10-30 \mathrm{~s}$ after stimulation.

\section{Results}

\section{The role of calcineurin in rapid and slow endocytosis at calyces}

The whole-cell capacitance was measured at the calyx in 7- to 10 -d-old rats. We induced slow and rapid endocytosis with 1 and 10 pulses of $20 \mathrm{~ms}$ depolarization (from -80 to $+10 \mathrm{mV}$, if not mentioned) at $10 \mathrm{~Hz}$, respectively (W. Wu et al., 2005; X. S. Wu et al., 2009). In control, at 4-10 min after whole-cell break in $(0.1 \%$ DMSO in pipette), a $20 \mathrm{~ms}$ depolarization induced a capacitance jump $\left(\Delta C_{\mathrm{m}}\right)$ of $462 \pm 31 \mathrm{fF}(n=12)$, followed by a monoexponential decay with a time constant $(\tau)$ of $18.6 \pm 1.0 \mathrm{~s}(n=$ $12)$ and an initial endocytosis rate $\left(\right.$ Rate $\left._{\text {decay }}\right)$ of $28 \pm 3 \mathrm{fF} / \mathrm{s}(n=$ 12 , Fig. $1 A$ ). Ten depolarizing pulses of $20 \mathrm{~ms}$ at $10 \mathrm{~Hz}$ induced a $\Delta C_{\mathrm{m}}$ of $1669 \pm 109 \mathrm{fF}(n=12)$, followed by a biexponential decay with $\tau$ of $2.8 \pm 0.3 \mathrm{~s}(44 \pm 4 \%)$ and $23.0 \pm 2.1 \mathrm{~s}(n=12$, Fig. $1 D)$, respectively. The Rate decay $_{\text {after }} 10$ depolarizing pulses was $270 \pm 20 \mathrm{fF} / \mathrm{s}(n=12$, Fig. $1 D)$, which reflected mostly $(>80 \%)$ the rapid component of endocytosis as demonstrated previously (W. Wu et al., 2005; X. S. Wu et al., 2009). This was confirmed in the present study, because the mean Rate decay $_{\text {of the }}$ rapid component of endocytosis was $\sim 262 \mathrm{fF} / \mathrm{s}$, as calculated from the ratio between its mean amplitude and mean time con$\operatorname{stant}\left(1669 \mathrm{fF}^{\star} 0.44 / 2.8 \mathrm{~s}=262 \mathrm{fF} / \mathrm{s}\right)$, whereas the mean Rate $_{\text {decay }}$ of the slow component of endocytosis was only $\sim 41 \mathrm{fF} / \mathrm{s}(=1669$ $\left.\mathrm{fF}^{\star} 0.56 / 23 \mathrm{~s}\right)$. In brief, these control experimental results were similar to previous reports (W. Wu et al., 2005; X. S. Wu et al., 2009).

We have previously shown that calcium influx triggers endocytosis and calmodulin blockers inhibited endocytosis (X. S. Wu et al., 2009). Consistent with this finding, replacing the extracellular calcium with barium, which barely activates calmodulin, also significantly inhibited endocytosis after 10 pulses of $20 \mathrm{~ms}$ depolarization at $10 \mathrm{~Hz}(n=5$, data not shown). To determine whether the calcium/calmodulin-activated calcineurin is involved in endocytosis, we measured endocytosis at 4-10 min after whole-cell break in with a pipette containing the calcineurin inhibitor cyclosporine A (CsA, $20 \mu \mathrm{M})$ or calcineurin autoinhibitory peptide $\left(\mathrm{CaN}_{457-482}, 150 \mu \mathrm{M}\right)$ (Oliveria et al., 2007). We found that CsA and $\mathrm{CaN}_{457-482}$, reduced the Rate decay $_{\text {after } 1}$ or 10 depolarizing pulses to only $\sim 24-32 \%$ of control (Fig. $1 A, B, D, E$, summarized in Fig. $1 C, F)$. We did not quantify the time constant, because we often did not observe any fast component of endocytosis, and slow endocytosis was often nearly blocked completely, which made quantification of the time constant impossible. Thus, throughout the study, we did not measure the time constant when endocytosis was inhibited.

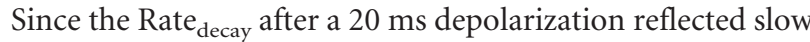
endocytosis, whereas $>80 \%$ of the Rate decay $_{\text {after the } 10 \text { pulse }}$ train was due to the rapid component of endocytosis, both calcineurin blockers significantly inhibited both slow and rapid endocytosis. The inhibition was not due to changes in calcium currents or exocytosis, because calcium currents did not change significantly, and $\Delta C_{\mathrm{m}}$ changed by $<20 \%$ (supplemental Information 1, available at www.jneurosci.org as supplemental material). These results suggest the involvement of calcineurin in both rapid and slow endocytosis.

Rapid and slow endocytosis can be induced not only by depolarizing pulses of $20 \mathrm{~ms}$, but also by trains of $1 \mathrm{~ms}$ depolarization that mimic action potential trains (Sun et al., 2002; W. Wu et al., 2005; X. S. Wu et al., 2009). For example, in the control condition with scrambled $\mathrm{CaN}_{457-482}(150 \mu \mathrm{M})$ in the pipette, 20 pulses of 1 ms depolarization to $+7 \mathrm{mV}$ at $200 \mathrm{~Hz}$ (AP-e), which mimicked a train of action potentials (Sun et al., 2002), induced a capacitance jump of $421 \pm 16 \mathrm{fF}(n=8)$, followed by a monoexponential decay with a time constant of $17.3 \pm 1.2 \mathrm{~s}(n=8)$ and a Rate $_{\text {decay }}$ of $30 \pm 1.6 \mathrm{fF} / \mathrm{s}(n=8$, Fig. $2 A)$. After 200 AP-e at 200 $\mathrm{Hz}$, the capacitance jump was $1331 \pm 85 \mathrm{fF}(n=8)$, followed by a biexponential decay with time constants of $2.3 \pm 0.3 \mathrm{~s}$ (46 \pm $3 \%, n=8)$ and $18.4 \pm 1.6 \mathrm{~s}(n=8)$, respectively (Fig. $2 B)$. The Rate $_{\text {decay }}$ after 200 AP-e was $252 \pm 23 \mathrm{fF} / \mathrm{s}(n=8$, Fig. 2 B). Thus, slow and rapid endocytosis induced by 20 and $200 \mathrm{AP}-\mathrm{e}$ at $200 \mathrm{~Hz}$ were similar to those induced by 1 and 10 pulses of $20 \mathrm{~ms}$ depolarization at $10 \mathrm{~Hz}$, respectively. Compared with the Rate $\mathrm{decay}_{\text {in }}$ the presence of scrambled $\mathrm{CaN}_{457-482}, \mathrm{CaN}_{457-482}(150 \mu \mathrm{M}$ in the pipette) significantly inhibited the Rate decay $_{\text {to }} 28 \pm 5 \%(n=8$, Fig. $2 \mathrm{~A}, \mathrm{C})$ after $20 \mathrm{AP}$-e at $200 \mathrm{~Hz}$, and to $34 \pm 11 \%(n=8$, Fig. $2 B, C)$ after $200 \mathrm{AP}-\mathrm{e}$ at $200 \mathrm{~Hz}(p<0.01)$. These results suggest that calcineurin blockers inhibit endocytosis not only after trains of $20 \mathrm{~ms}$ depolarization, but also after trains of $1 \mathrm{~ms}$ depolarization that mimic action potential trains.

The calcineurin blocker specificity is often a concern that might discount the significance of pharmacological experiments. To address this issue, we used 7- to 10-d-old mice lacking cal- 
A

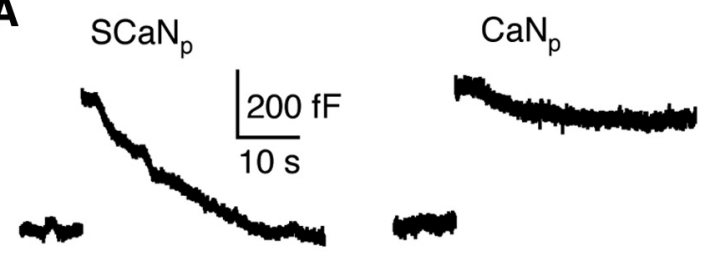

B
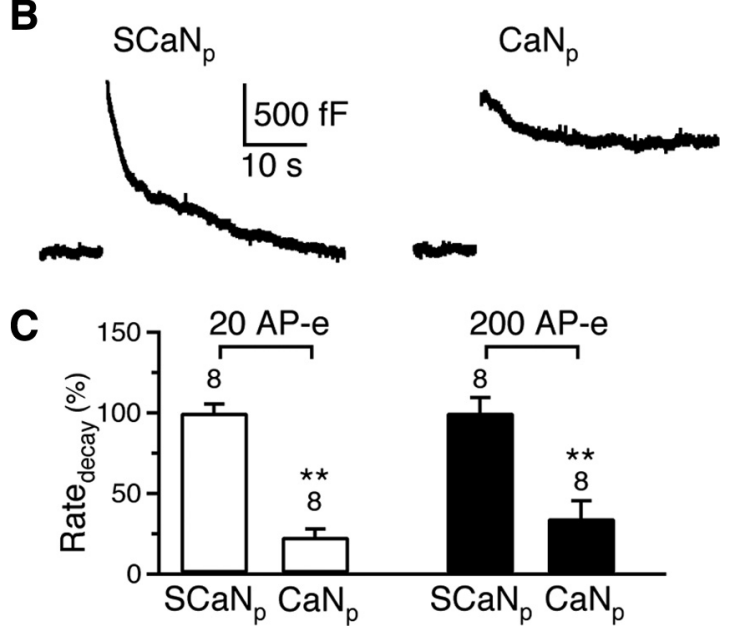

Figure 2. $\mathrm{CaN}_{457-482}$ inhibits rapid and slow endocytosis induced by AP-e trains at the calyx. $A$, Two sampled $C_{\mathrm{m}}$ traces showing endocytosis induced by $20 \mathrm{AP}$-e at $200 \mathrm{~Hz}$ with a pipette containing either scrambled $\mathrm{CaN}_{457-482}\left(\mathrm{SCaN}_{\mathrm{p}}, 150 \mu \mathrm{M}\right)$ or $\mathrm{CaN}_{457-482}\left(\mathrm{CaN}_{\mathrm{p}}, 150 \mu \mathrm{M}\right) . \boldsymbol{B}$, Similar to A, except that the stimulus was $200 \mathrm{AP}$-e at $200 \mathrm{~Hz}$, which induced both rapid and slow endocytosis in control $\left(\mathrm{SCaN}_{\mathrm{p}}\right)$. C, Comparison of the Rate ${ }_{\text {decay }}$ in the presence of $\mathrm{SCaN}_{\mathrm{p}}$ $(150 \mu \mathrm{M})$ or $\mathrm{CaN}_{\mathrm{p}}(150 \mu \mathrm{m})$. The stimulus was either 20 (open bars) or 200 (solid bars) AP-e at $200 \mathrm{~Hz}$. Data for open and solid bars were normalized to the mean value of open and solid SCaN group, respectively.

cineurin $\mathrm{A}_{\alpha}$ or $\mathrm{A}_{\beta}$ subunit. Calcineurin is composed of a catalytic $\mathrm{A}$ and a regulatory $\mathrm{B}$ subunit. Among three isoforms of the $\mathrm{A}$ subunit, $\mathrm{A}_{\alpha}$ and $\mathrm{A}_{\beta}$ are expressed in the brain (Rusnak and Mertz, 2000). $\mathrm{A}_{\alpha}^{-1-}$ or $\mathrm{A}_{\beta}^{-1-}$ mice had been generated (Zhang et al., 1996; Bueno et al., 2002), from which we could not generate double knock-out mice $\left(\mathrm{A}_{\alpha}^{-1-} \mathrm{A}_{\beta}^{-1-}\right)$, likely because they die in the embryonic stage as observed in calcineurin $\mathrm{B}$ knock-out (Chang et al., 2004).

In wild-type (WT) mice, the Rate decay $_{\text {was }} 28 \pm 4 \mathrm{fF} / \mathrm{s}$ ( $n=10$, Fig. $3 A)$ and $157 \pm 26 \mathrm{fF} / \mathrm{s}(n=10$, Fig. $3 B)$ after 1 and 10 depolarizing pulses at $10 \mathrm{~Hz}$, respectively. Similar to rat calyces

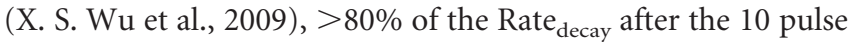
train was due to rapid endocytosis. Compared with WT mice, the Rate $_{\text {decay }}$ after 1 (Fig. 3A) or 10 depolarizing pulses (Fig. $3 B$ ) was reduced by $>50 \%$ in $\mathrm{A}_{\alpha}^{-1-}$ mice $(p<0.01)$, but did not change significantly in $\mathrm{A}_{\beta}^{-1-}$ mice $(p>0.5)$. The Rate $\mathrm{decay}_{\text {deduction in }}$ $\mathrm{A}_{\alpha}^{-1-}$ mice was not due to changes in calcium currents or $\Delta C_{\mathrm{m}}$ (supplemental Information 2, available at www.jneurosci.org as supplemental material). Thus, calcineurin $\mathrm{A}_{\alpha}$, but not $\mathrm{A}_{\beta}$ subunit, is involved in mediating both rapid and slow endocytosis at calyces.

\section{Endocytosis at hippocampal synapses}

The calyx-type synapse is much larger than the conventional synapse. Whether our findings at calyces apply to conventional synapses is unclear. We addressed this issue at cultured hippocampal synapses by examining the roles of calcium, calmodulin, and calcineurin. SynaptopHluorin $(\mathrm{SpH})$ was transfected to cultured rat hippocampal synapses (Sankaranarayanan and Ryan, 2000). Field electrical stimulation $(20 \mathrm{~mA}, 1 \mathrm{~ms})$ was applied to induce action potentials. In control, a $20 \mathrm{~Hz}$ stimulation train for $10 \mathrm{~s}$ (Train ${ }_{10}$ ) caused exocytosis and thus a fluorescence increase $\left(\Delta F_{\text {peak }}\right)$ of $35 \pm 5 \%$ of the baseline intensity $(n=7$ experiments, each experiment contained $\sim 10-30$ boutons, Fig. $4 A$, left). The fluorescence increase was followed by a mono-exponential decay, because of $\mathrm{SpH}$ endocytosis and vesicle reacidification. The decay reflects mostly endocytosis, because endocytosis usually takes much longer than $10 \mathrm{~s}$, whereas reacidification takes only 3-4 s (Atluri and Ryan, 2006; Granseth et al., 2006). The rate of the initial fluorescence decay ( Rate $\left._{\text {decay }}\right)$ was $1.06 \pm 0.18 \% / \mathrm{s}(n=7$, fluorescence intensity normalized to baseline). The decay $\tau$ was $41.9 \pm 2.4 \mathrm{~s}(n=7$, Fig. $4 A$, left $)$. The fluorescence increase at $100 \mathrm{~s}$ after stimulation $\left(\Delta F_{100 \mathrm{~s}}\right)$ was $-1 \pm 11 \%(n=7)$ of $\Delta F_{\text {peak }}$, indicating completed endocytosis (Fig. $4 A$, left). Compared with Train $_{10 \mathrm{~s}}$, a $20 \mathrm{~Hz}$ train for $2 \mathrm{~s}$ ( Train $_{2 \mathrm{~s}}$ ) induced a smaller $\Delta F_{\text {peak }}$ (16 $\pm 5 \%$ of the baseline), a smaller decay $\tau(20.9 \pm 2.1 \mathrm{~s}$ ), but only a slightly smaller Rate decay $(0.86 \pm 0.09 \% / \mathrm{s})$, and a similar $\Delta F_{100 \mathrm{~s}}$ $\left(-6 \pm 7 \%\right.$ of $\Delta F_{\text {peak }}, n=6$, Fig. $4 A$, right $)$.

\section{The role of calcium at hippocampal synapses}

An early study showed that decreasing the extracellular calcium concentration $\left(\left[\mathrm{Ca}^{2+}\right]_{\mathrm{o}}\right)$ to $0.75 \mathrm{~mm}$ or applying the calcium buffer EGTA-AM reduced the Rate decay $_{\text {by severalfold (Sankara- }}$ narayanan and Ryan, 2001). Given that the $\left[\mathrm{Ca}^{2+}\right]_{\mathrm{o}}$ did not affect vesicle reacidification, it was concluded that calcium influx regulates endocytosis. If calcium influx not only regulates endocytosis, but also initiates endocytosis, further reducing the $\left[\mathrm{Ca}^{2+}\right]_{\mathrm{o}}$ should nearly abolish endocytosis as has been shown at calyces (Hosoi et al., 2009; X. S. Wu et al., 2009). Indeed, at $0.25 \mathrm{~mm}$ $\left[\mathrm{Ca}^{2+}\right]_{\mathrm{o}}$, Train $10 \mathrm{~s}$ induced a Rate decay $(0.20 \pm 0.04 \% / \mathrm{s}, n=4)$ much smaller than that at $2 \mathrm{~mm}\left[\mathrm{Ca}^{2+}\right]_{\mathrm{o}}$ by $\operatorname{Train}_{10 \mathrm{~s}}$ or $\operatorname{Train}_{2 \mathrm{~s}}$ $(p<0.01)$, and induced a $\Delta F_{100 \text { s }}$ as large as $73 \pm 7 \%(n=4)$ of $\Delta F_{\text {peak }}($ Fig. $4 B)$. At $0.1 \mathrm{~mm}\left[\mathrm{Ca}^{2+}\right]_{\mathrm{o}}$, Train ${ }_{10 \text { s }}$ could not induce a detectable $\Delta F_{\text {peak }}$. However, a $10 \mathrm{~s}$ train at $100 \mathrm{~Hz}$ induced a $\Delta F_{\text {peak }}(22 \pm 5 \%)$ between those induced by $\operatorname{Train}_{2 \mathrm{~s}}$ and $\operatorname{Train}_{10 \mathrm{~s}}$ at $2 \mathrm{mM}\left[\mathrm{Ca}^{2+}\right]_{\mathrm{o}}$, but a Rate decay $(0.07 \pm 0.04 \% / \mathrm{s}$, Fig. $4 \mathrm{C}) 12-$ to 14-fold smaller than that induced by Train $_{2 \mathrm{~s}}$ or $\operatorname{Train}_{10 \mathrm{~s}}$ at $2 \mathrm{mM}$ $\left[\mathrm{Ca}^{2+}\right]_{\mathrm{o}}$, and a $\Delta F_{100 \mathrm{~s}}$ as large as $80 \pm 10 \%$ of $\Delta F_{\text {peak }}(n=5)$. At $2 \mathrm{~mm}\left[\mathrm{Ca}^{2+}\right]_{\mathrm{o}}$, this $100 \mathrm{~Hz}$ train induced a much larger $\Delta F_{\text {peak }}$ $(104 \pm 5 \%)$, a $\operatorname{Rate}_{\text {decay }}(1.35 \pm 0.06 \% / \mathrm{s}) \sim 20$ times higher than that at $0.1 \mathrm{~mm}\left[\mathrm{Ca}^{2+}\right]_{\mathrm{o}}$, and a much smaller $\Delta F_{100 \mathrm{~s}}(14 \pm 3 \%$ of $\Delta F_{\text {peak }}, n=4$, Fig. $\left.4 D\right)$. Clearly, decreasing the $\left[\mathrm{Ca}^{2+}\right]_{\mathrm{o}}$ from 2 to $0.1 \mathrm{~mm}$ reduced the Rate decay to nearly 0 (Fig. $4 E$ ), and significantly increased $\Delta F_{100 \mathrm{~s}}$ (Fig. $4 F$ ). These results suggest an essential role of calcium in controlling the rate of endocytosis, similar to results observed at the calyx of Held (Hosoi et al., 2009; X. S. Wu et al., 2009).

At $2 \mathrm{~mm}\left[\mathrm{Ca}^{2+}\right]_{\mathrm{o}}$, as the $\Delta F_{\text {peak }}$ increased to $\sim 16 \%$ (induced by $\operatorname{Train}_{2 \mathrm{~s}}$ ), the Rate ${ }_{\text {decay }}$ increased to $\sim 0.86 \% / \mathrm{s}$ (Fig. $4 G$, solid square). Further increasing the $\Delta F_{\text {peak }}$ to $\sim 104 \%$ (induced by the $100 \mathrm{~Hz}$ train), which was $\sim 6.5$-fold larger than that $(16 \%)$ induced by Train $_{2 \mathrm{~s}}$, only increased the Rate decay $_{\text {en }}$ to $1.35 \% / \mathrm{s}$ (Fig. $4 G$, solid triangle). Thus, the endocytosis capacity may be partially saturated at a $\Delta F_{\text {peak }}$ of $\geq 16 \%$ (Sankaranarayanan and Ryan, 2001; Balaji et al., 2008). The increase in the Rate decay $_{\text {might }}$ be due to an increase of the $\Delta F_{\text {peak }}$ and/or an increase of the frequency of stimulation. However, the decrease of the Rate decay at $0.1-0.25 \mathrm{~mm}\left[\mathrm{Ca}^{2+}\right]_{0}$ was independent of either of these changes (Fig. 4G, comparing open and solid symbols). In particular, the Rate decay $_{\text {at }} 0.1-0.25 \mathrm{~mm}\left[\mathrm{Ca}^{2+}\right]_{\mathrm{o}}$ (Fig. $4 G$, open symbols) was much smaller than that at $2 \mathrm{mM}\left[\mathrm{Ca}^{2+}\right]_{0}$ at similar $\Delta F_{\text {peak }}$ values (Fig. $4 G$, solid square and circle). These results 
A
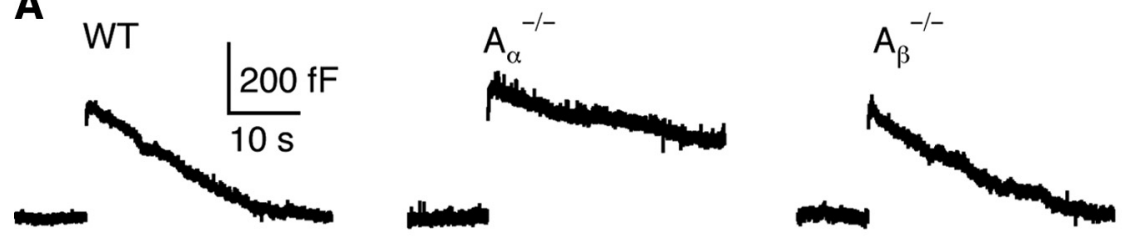

B

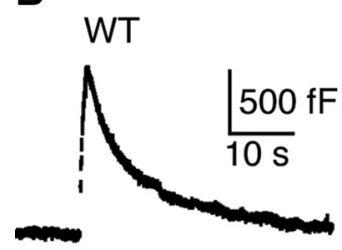

$\mathrm{A}_{\alpha}^{-/-}$

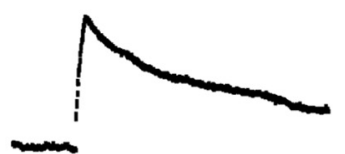

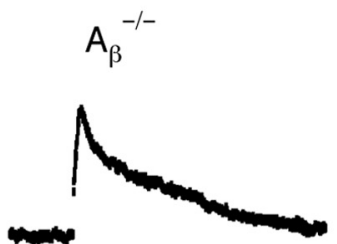
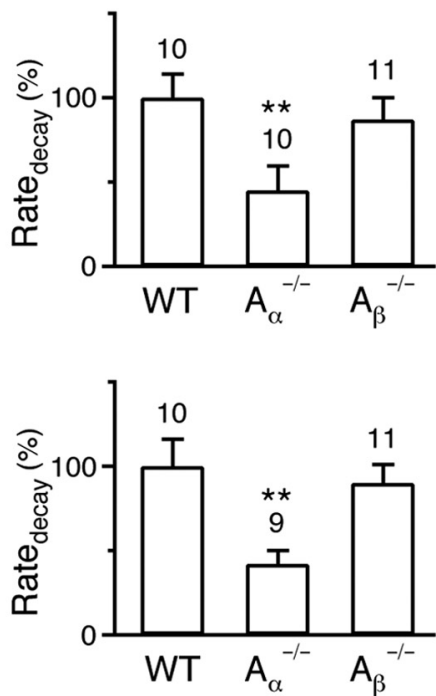

Figure 3. Knock-out of calcineurin $\mathrm{A}_{\alpha}$ subunit inhibits rapid and slow endocytosis at the calyx. $A$, Left, Sampled $C_{m}$ induced by a 20 ms depolarization in a WT mouse, a calcineurin $\mathrm{A}_{\alpha}^{-1-}$ mouse

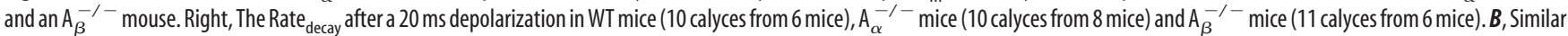
to $A$, except that the stimulus was 10 pulses of 20 ms depolarization at $10 \mathrm{~Hz}$.

suggest that the reduced calcium influx at low $\left[\mathrm{Ca}^{2+}\right]_{0}$, but not the change in the amount of exocytosis, decreased the Rate $_{\text {decay. }}$.

The role of calmodulin at

hippocampal synapses

In the presence of a calmodulin blocker, calmidazolium (CMDZ, $10 \mu \mathrm{M}$ in the bath, 5-10 min), the Rate decay $_{\text {after Train }}$ Tr s $_{\text {s }}(0.28 \pm$ $0.09 \% / \mathrm{s}, n=7$ ) was much smaller than that $(0.86-1.06 \% / \mathrm{s})$ after Train ${ }_{10 \mathrm{~s}}$ or Train $_{2 \mathrm{~s}}$ in control $(p<0.01)$, and the $\Delta F_{100 \mathrm{~s}}(79 \pm$ $14 \%$ of $\left.\Delta F_{\text {peak }}, n=7\right)$ was much larger (Fig. 5A). The block of the $\mathrm{SpH}$ fluorescence decay was not due to inhibition of vesicle reacidification (supplemental Information 3, available at www.jneurosci. org as supplemental material). Thus, CMDZ inhibits endocytosis at hippocampal synapses.

The $\Delta F_{\text {peak }}$ induced by $\operatorname{Train}_{10 \text { s }}$ in the presence of CMDZ was smaller than that induced by Train $10 \mathrm{~s}$ in control, but larger than that induced by Train $_{2 \mathrm{~s}}$ in control (Fig. $5 A$ ). The reduction of the $\Delta F_{\text {peak }}$ was not responsible for the decrease of the Rate $_{\text {decay }}$, because Train ${ }_{2}$ in control induced a smaller $\Delta F_{\text {peak }}$, but a much larger Rate $_{\text {decay }}$ than that induced by Train ${ }_{10 \mathrm{~s}}$ in the presence of CMDZ (Fig. 5A). The reduction of the $\Delta F_{\text {peak }}$ by CMDZ was consistent with the finding that calmodulin promotes vesicle mobilization from the reserve pool to the readily releasable pool (Sakaba and Neher, 2001), likely by initiating endocytosis that clears the released vesicle proteins from the release site (X. S. Wu et al., 2009).

CMDZ might not be specific to only calmodulin. To address this issue, we used
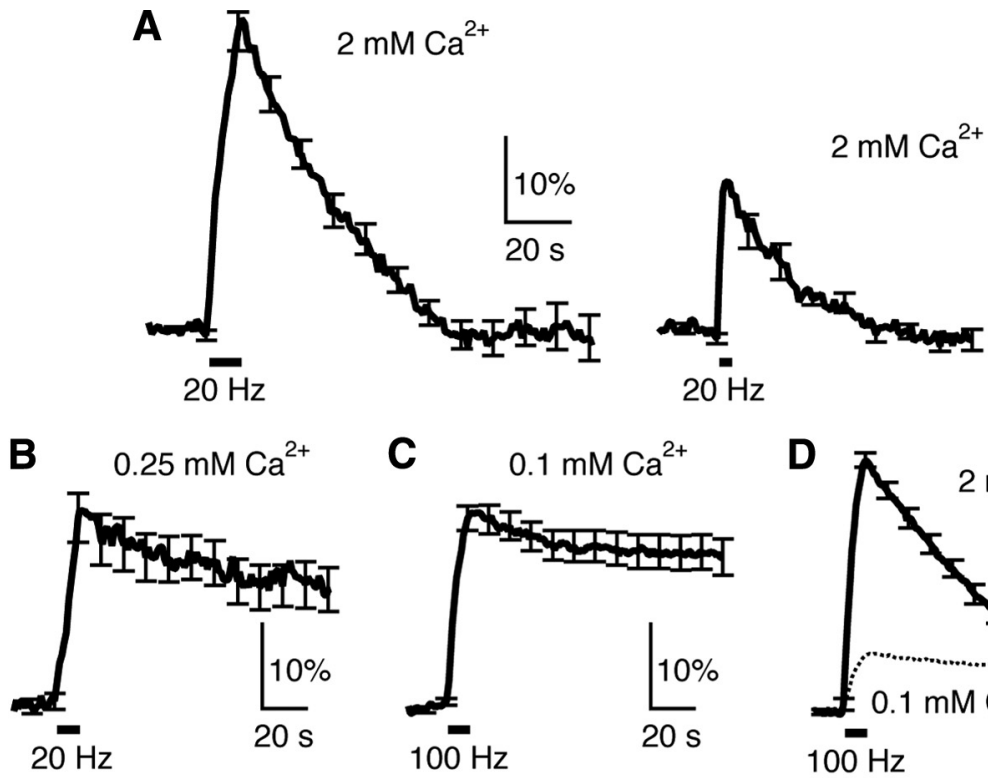

C $\quad 0.1 \mathrm{mM} \mathrm{Ca}^{2+}$

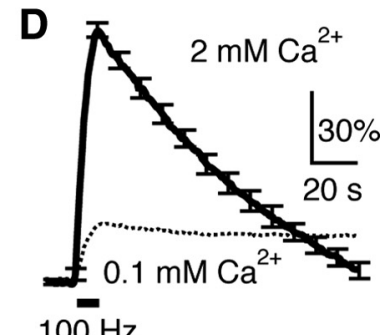

E

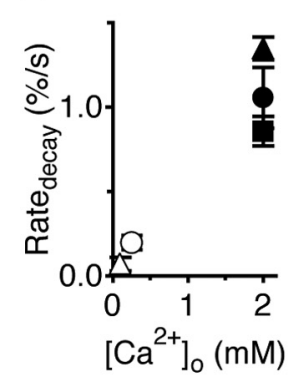

$\mathbf{F}$

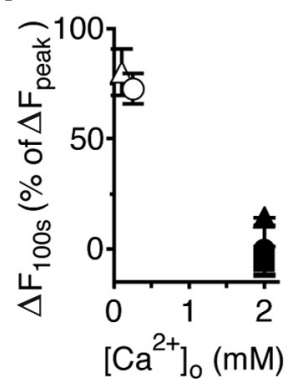

G

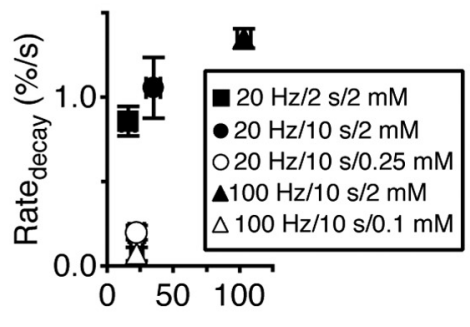

$\Delta \mathrm{F}_{\text {peak }}(\%$ of baseline)
Figure 4. Decrease of the $\left[\mathrm{Ca}^{2+}\right]_{0}$ nearly abolishes endocytosis at hippocampal synapses. $\boldsymbol{A}$, The SpH signal induced by $\operatorname{Train}_{10 s}$ ( $n=7$ experiments, left) or $\operatorname{Train}_{2 \mathrm{~s}}$ ( $n=6$, right) at $2 \mathrm{~mm}\left[\mathrm{Ca}^{2+}\right]_{0}$. Data were plotted as mean \pm SE. The SE was plotted every 10 s (applies to Figs. $4-7)$. $\boldsymbol{B}$, The SpH signal induced by $\operatorname{Train}_{10 \mathrm{~s}}$ at $0.25 \mathrm{~mm}\left[\mathrm{Ca}^{2+}\right]_{0}(n=4)$. $\boldsymbol{C}$, The SpH signal induced by a $100 \mathrm{~Hz}$ train for $10 \mathrm{~s}$ at $0.1 \mathrm{~mm}\left[\mathrm{Ca}^{2+}\right]_{0}(n=5)$. D, The SpH signal induced by a $100 \mathrm{~Hz}$ train for $10 \mathrm{~s}$ at $2 \mathrm{~mm}\left[\mathrm{Ca}^{2+}\right]_{0}(n=4)$. The

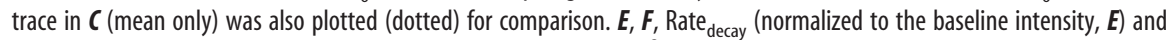
$\Delta \boldsymbol{F}_{100 \mathrm{~s}}(\boldsymbol{F})$ induced by stimuli listed in $\boldsymbol{A}-\boldsymbol{D}$ are plotted versus the $\left[\mathrm{Ca}^{2+}\right]_{0}$. Symbols in $\boldsymbol{G}$ apply to $\boldsymbol{E}-\boldsymbol{G}$. $\boldsymbol{G}$, Rate ${ }_{\text {decay }}$ induced by stimuli listed in $\boldsymbol{A}-\boldsymbol{D}$ is plotted versus the $\Delta F_{\text {peak }}$ (normalized to the baseline). 
A
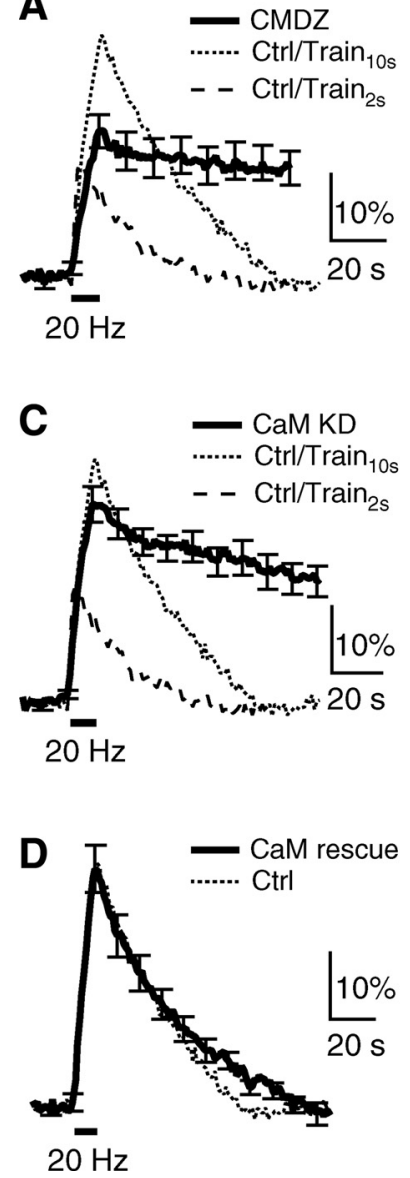

B

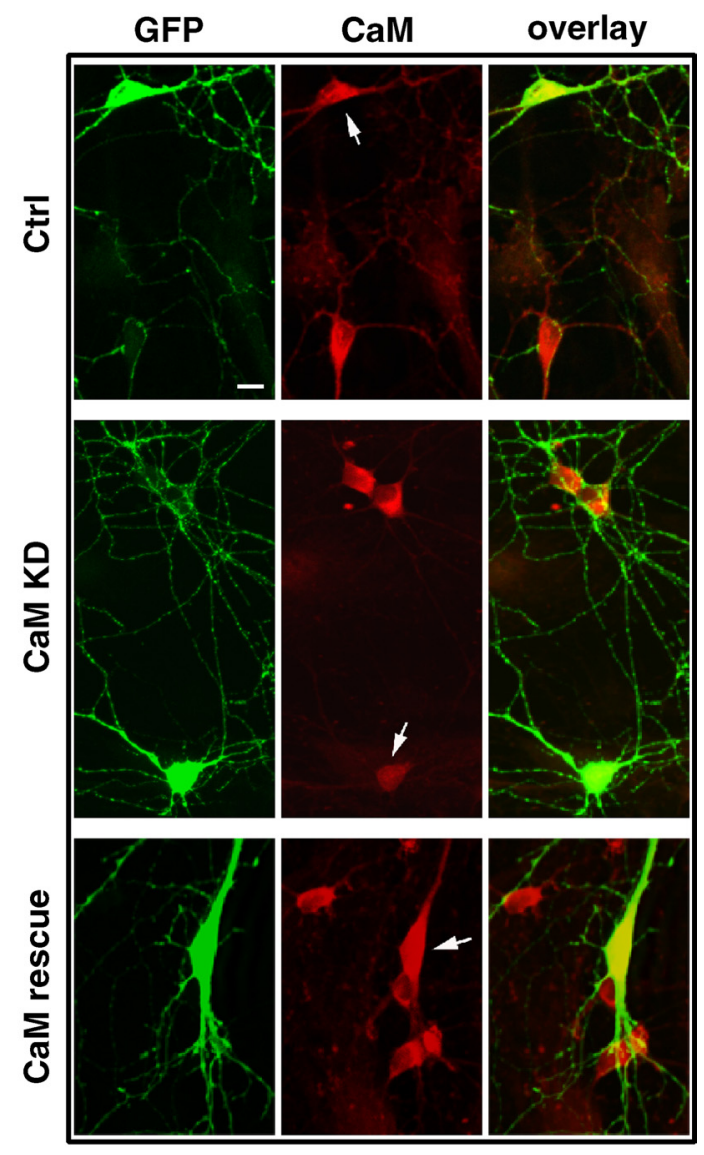

Figure 5. The role of calmodulin in slow endocytosis at hippocampal synapses. $\boldsymbol{A}$, The SpH signal induced by $\mathrm{Train}_{10 \mathrm{~s}}$ in the presence of $10 \mu \mathrm{m}$ calmidazolium (CMDZ, 5-10 min, bath application). For comparison, the mean SpH signal induced by Train ${ }_{10} \mathrm{~s}$ (dotted) and Train ${ }_{2 s}$ (dash) in control are also shown. $\boldsymbol{B}$, Staining of an antibody against green fluorescence protein (GFP), which also recognized $\mathrm{SpH}$ (left, green), and an antibody against calmodulin (middle, red) at hippocampal cultures transfected with SpH along (upper, $(\mathrm{trl})$ ), SpH and calmodulin shRNA (middle, (aM KD), or SpH and a plasmid containing calmodulin shRNA and shRNA-resistant calmodulin (lower, CaM rescue). The green and red images are superimposed in the right. Arrows indicate transfected neurons. The soma of a neuron transfected with calmodulin shRNA showed a much lower calmodulin staining compared with un-transfected neurons (middle, CaM KD). Scale bar, $10 \mu \mathrm{m}$. C, The SpH signal induced by Train 10 s in hippocampal boutons transfected with calmodulin shRNA ( $n=15$ experiments). The mean SpH signal induced by Train ${ }_{10 \text { s }}$ (dotted) and Train 2 $s$ (dash) in control are also shown. D, The SpH signal induced by Train $_{10}$ s in boutons transfected with CaM rescue plasmid $(n=9)$. The mean SpH signal induced by $\operatorname{Train}_{10 \text { s }}$ (dotted) in control is also shown.

a calmodulin shRNA that can knock down calmodulin expression by $\sim 70 \%$ in cultured cortical neurons (Pang et al., 2010). Transfection of this shRNA to PC12 cells reduced calmodulin to $32 \pm 6 \%(n=6)$ of control (supplemental Information 4, available at www.jneurosci.org as supplemental material). Cotransfection of calmodulin shRNA and $\mathrm{SpH}$ reduced calmodulin in the soma of rat hippocampal neurons to $30 \pm 2 \%$ ( $n=10$ neurons from 3 transfections, $p<0.01$ ) of that in neighbor un-transfected neurons (Fig. $5 B$, middle). In transfected neurons, Train 10 s induced a Rate decay $(0.38 \pm$ $0.04 \% / \mathrm{s}, n=15)$ much slower than that $(0.86-1.06 \% / \mathrm{s})$ induced by Train $_{10}$ or Train ${ }_{2 s}$ in control $(p<0.01)$, and a much larger $\Delta F_{100 \mathrm{~s}}$ $\left(61 \pm 8 \%\right.$ of $\Delta F_{\text {peak }}, n=15$, Fig. $\left.5 C\right)$, suggesting an inhibition of endocytosis similar to that caused by CMDZ. The $\Delta F_{\text {peak }}$ induced by $\operatorname{Train}_{10 \mathrm{~s}}$ was also slightly reduced compared with the control (Fig. $5 C$ ), consistent with the effects of CMDZ in blocking vesicle mobilization to the readily releasable pool (Fig. $5 \mathrm{~A}$ ) (Sakaba and Neher, 2001).

The decrease of the calmodulin level in neurons cotransfected with calmodulin shRNA and $\mathrm{SpH}$ (Fig. 5B, middle) was not due to transfection of $\mathrm{SpH}$. This was because transfection of SpH along did not affect the calmodulin level in the soma, compared with the neighbor un-transfected neurons (103 $\pm 3 \%, n=7$ neurons, 2 transfections, $p>0.1$, Fig. $5 B$, top). In neurons cotransfected with $\mathrm{SpH}$ and a plasmid containing both calmodulin shRNA and shRNAresistant calmodulin, calmodulin was over rescued to $163 \pm 4 \%(n=11$ neurons from 3 transfections, $p<0.01$ ) of that in un-transfected neurons (Fig. $5 B$, bottom), and the $\operatorname{Rate}_{\text {decay }}(1.02 \pm 0.06 \% /$ s), $\Delta F_{100 \mathrm{~s}}\left(-3 \pm 4 \%\right.$ of $\left.\Delta F_{\text {peak }}\right)$ and $\Delta F_{\text {peak }}$ (36 $\pm 4 \%, n=9)$ induced by $\operatorname{Train}_{10 \mathrm{~s}}$ were similar to control $(p>0.18$, Fig. $5 D)$. Transfection of this plasmid to PC12 cells also increased the calmodulin expression to $152 \pm 5 \%$ of control $(n=3$, supplemental Information 4, available at www.jneurosci.org as supplemental material) (see also Pang et al., 2010). These results suggest that inhibition of endocytosis by calmodulin shRNA was not due to off-target shRNA effects. We concluded that the physiological level of calmodulin is sufficient and critical in mediating normal endocytosis. This result, together with a recent finding that calmodulin may enhance the release probability by activation of CaMKII at hippocampal synapses (Pang et al., 2010), suggest that calmodulin is important not only for endocytosis, but also for exocytosis.

\section{The role of calcineurin at hippocampal synapses}

In the presence of the calcineurin blocker cyclosporin A (CsA, $20 \mu \mathrm{M}$ in the bath, 5-10 min), Train ${ }_{10}$ s induced a $\Delta F_{\text {peak }}$ $(66 \pm 8 \%, n=13)$ nearly two times the control, but a Rate decay $(0.72 \pm 0.14 \% / \mathrm{s}$, $n=13)$ smaller than the control (1.06 \pm $0.18 \% / \mathrm{s}, n=7, p<0.05)$, and a much larger $\Delta F_{100 \mathrm{~s}}(62 \pm 8 \%$, $n=13$, Fig. $6 A)$. The initial rate of endocytosis $\left(\right.$ Rate $\left._{\text {decay }}\right)$ increases as the amount of exocytosis $\left(\Delta F_{\text {peak }}\right)$ increases (Balaji et al., 2008) until the latter reaches the endocytic capacity (Wu and Betz, 1996; Sankaranarayanan and Ryan, 2000; Sun et al., 2002) (see also Fig. $4 G$, solid symbols). Thus, an increase of the $\Delta F_{\text {peak }}$

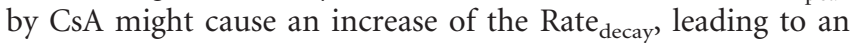
underestimate of the inhibition of Rate decay by CsA. To examine this possibility, we divided the CsA experiments into two groups with $\Delta F_{\text {peak }}$ smaller or larger than $50 \%$ of the baseline. The reason we used $50 \%$ to divide the data was that the group with a smaller $\Delta F_{\text {peak }}$ had a $\Delta F_{\text {peak }}(40 \pm 3 \%, n=5)$ similar to that induced by Train $_{10 \mathrm{~s}}$ in control. This group had $\sim 7$-fold smaller Rate ${ }_{\text {decay }}$ $(0.16 \pm 0.04 \% / s, p<0.01)$, and a much larger $\Delta F_{100 \mathrm{~s}}(84 \pm 13 \%$; Fig. $6 B$, left, comparing solid and dotted traces). The group with a larger $\Delta F_{\text {peak }}$ had a mean $\Delta F_{\text {peak }}(82 \pm 8 \%, n=8)$ close to that induced by the $100 \mathrm{~Hz}$ train for $10 \mathrm{~s}$ in control $(103 \pm 5 \%, n=4)$, but had a smaller Rate decay $(0.85 \pm 0.06 \% / s, n=8, p<0.01)$ and a larger $\Delta F_{100 \mathrm{~s}}(48 \pm 5 \%, n=8, p<0.01)$ compared with that in- 
A

B

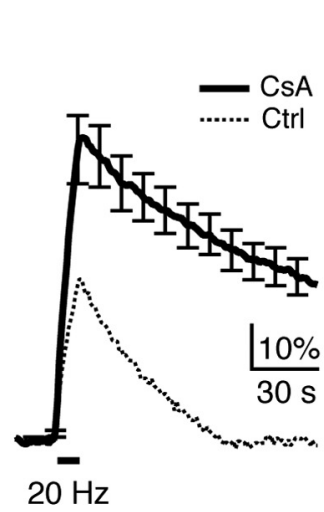

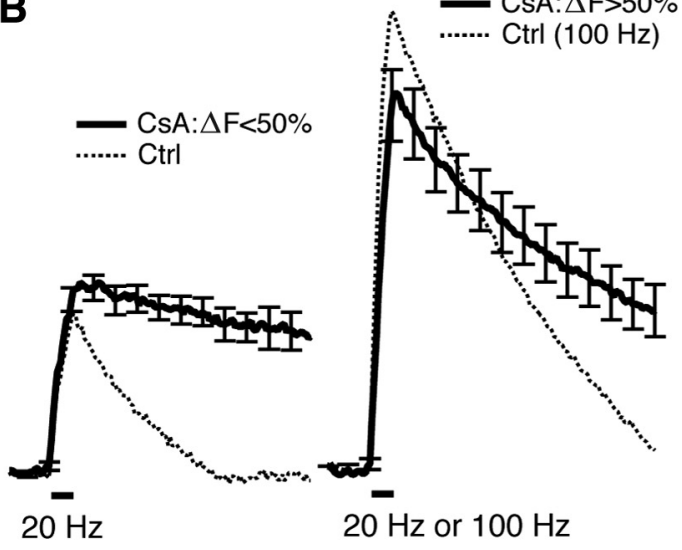

C
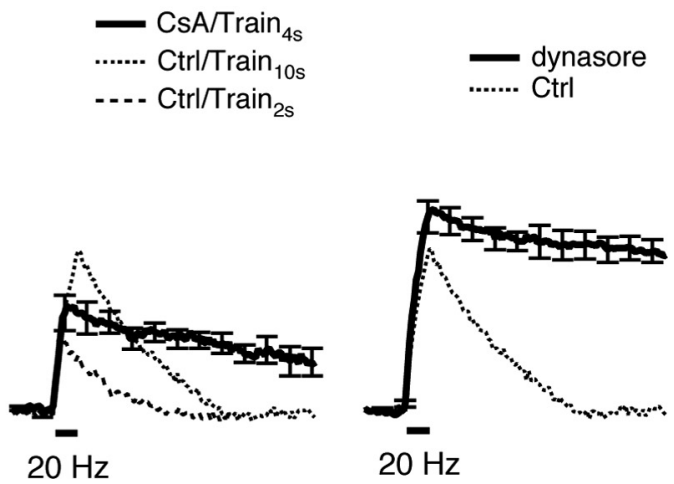

Figure 6. Calcineurin inhibitor (sA inhibits slow endocytosis at hippocampal synapses. $\boldsymbol{A}$, The SpH signal induced by $\operatorname{Train}_{10}$ s in the presence of $20 \mu \mathrm{m}$ CsA ( $n=13$, solid). For comparison, the mean SpH signal induced by Train ${ }_{10}$ in control is also plotted (dotted). $\boldsymbol{B}$, The CsA experiments (solid trace in $\boldsymbol{A}$ ) were divided into two groups depending on whether the $\Delta F_{\text {peak }}$ is less than (left, $n=$ 5) or lager than (right, $n=8$ ) $50 \%$ of the baseline (solid). The mean $\mathrm{SpH}$ signal induced by Train $_{10}$ s in control is also plotted in the left (dotted), whereas the mean $\mathrm{SpH}$ signal induced by a $100 \mathrm{~Hz}$ train for 10 s in control is plotted in the right (dotted). C, The SpH signal induced by a $20 \mathrm{~Hz}$ train for $4 \mathrm{~s}$ (Train ${ }_{4 \mathrm{~s}}$ ) in the presence of $20 \mu \mathrm{m}$ (sA ( $n=6$, solid). For comparison, the mean SpH signals

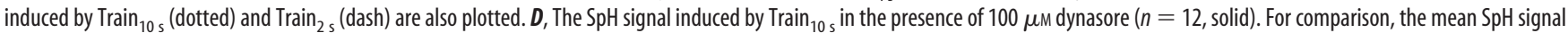
induced by Train $_{10}$ s in control is also plotted (dotted).

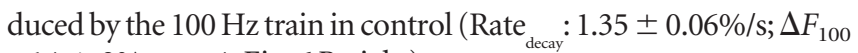
s: $14 \pm 3 \% ; n=4$, Fig. $6 B$, right).

Clearly, CsA was more effective in blocking endocytosis at smaller $\Delta F_{\text {peak }}$ (Fig. $6 B$ ). Consistent with this result, a 4 s stimulation train at $20 \mathrm{~Hz}$ in the presence of CsA induced a $\Delta F_{\text {peak }}$ (23 $\pm 4 \%, n=6)$ between those induced by Train $_{10 \mathrm{~s}}$ and Train $_{2 \mathrm{~s}}$ in control, but an $\sim 3$ - to 4 -fold smaller Rate decay $(0.26 \pm$ $0.08 \% / \mathrm{s})$, and a much larger $\Delta F_{100 \mathrm{~s}}(63 \pm 11 \%$, Fig. $6 C)$ than those induced by $\operatorname{Train}_{10 \mathrm{~s}}$ or $\operatorname{Train}_{2 \mathrm{~s}}$ in control. Large $\Delta F_{\text {peak }}$ may force the endocytic machinery to operate at near maximal capacity (Sankaranarayanan and Ryan, 2000), at which inhibition could be more difficult. These results, and the observation that CsA did not inhibit vesicle reacidification (supplemental Information 5, available at www.jneurosci.org as supplemental material), suggest that CsA significantly inhibited endocytosis.

The increase of $\Delta F_{\text {peak }}$ by CsA (Fig. $6 A$ ) could be due to a block of endocytosis and/or an increase of release. To distinguish these possibilities, a dynamin inhibitor, dynasore $(100 \mu \mathrm{M})$ was applied to the bath for 5-10 min, which essentially blocked endocytosis after Train $_{10 \mathrm{~s}}$ (Fig. 6D) (Newton et al., 2006). In this condition, Train $10 \mathrm{~s}$ induced a $\Delta F_{\text {peak }}(44 \pm 4 \%, n=12$, Fig. $6 D)$ higher than that $(35 \pm 5 \%, n=7, p<0.05)$ in control, but smaller than that $(66 \pm$ $8 \%, n=13, p<0.05$, Fig. $6 A)$ in the presence of CsA. These results suggest that CsA may also increase release, consistent with previous reports that block of calcineurin increases transmitter release by an as yet unidentified mechanism (Sihra et al., 1995; Lin and Lin-Shiau, 1999; Chi et al., 2003).

Next, we studied endocytosis in hippocampal cultures of calcineurin $\mathrm{A}_{\beta}^{-1-}$ or $\mathrm{A}_{\alpha}^{-1-}$ mice where the block of calcineurin function is more specific. In WT mice, Train ${ }_{10 \text { s }}$ induced a $\Delta F_{\text {peak }}$ of $36 \pm 3 \%$, a Rate decay $_{\text {of }} 0.95 \pm 0.05 \% / \mathrm{s}$, and a $\Delta F_{100 \text { s }}$ of $3 \pm 7 \%$ $(n=4)$, which were nearly the same as those obtained in control rats (comparing the dotted trace in Fig. 7A, 6A). In $\mathrm{A}_{\beta}^{-1-}$ mice, Train $_{10 \mathrm{~s}}$ induced a $\Delta F_{\text {peak }}(79 \pm 8 \%, n=21)$ much larger than the WT $(p<0.01$, Fig. $7 A)$, which was similar to the effects of CsA (Fig. 6A). Similar to the CsA experiments (Fig. 6B), we divided the data into two groups depending on whether the $\Delta F_{\text {peak }}$ was smaller or larger than $50 \%$ (Fig. $7 B$ ). The group with a smaller $\Delta F_{\text {peak }}$ had a $\Delta F_{\text {peak }}(40 \pm 4 \%, n=5)$ similar to that induced by Train $_{10}$ s in WT, but an $\sim 3$-fold smaller Rate decay
$(0.33 \pm 0.02 \% / \mathrm{s}, n=5, p<0.01)$, and a much larger $\Delta F_{100 \mathrm{~s}}$ ( $66 \pm 9 \%, n=5, p<0.01$; Fig. $7 B$, left). The group with a larger $\Delta F_{\text {peak }}$ had a mean $\Delta F_{\text {peak }}(91 \pm 8 \%, n=16)$ close to that induced by the $100 \mathrm{~Hz}$ train for $10 \mathrm{~s}$ in WT $(106 \pm 8 \%, n=8)$, but a Rate $_{\text {decay }}(0.91 \pm 0.05 \% / \mathrm{s}, n=16)$ smaller than that induced by the $100 \mathrm{~Hz}$ train in WT $(1.48 \pm 0.02 \% / \mathrm{s}, n=8, p<0.01)$, and a much larger $\Delta F_{100 \mathrm{~s}}\left(\mathrm{~A}_{\beta}^{-l-}: 53 \pm 6 \%, n=16\right.$; WT: $23 \pm 5 \%, n=$ $8, p<0.01$; Fig. $7 B$, right).

Similar to the effect of CsA, knock-out of calcineurin $\mathrm{A}_{\beta}$ was more effective in blocking endocytosis at smaller $\Delta F_{\text {peak }}$ (Fig. $7 B$ ). Consistent with this result, a $4 \mathrm{~s}$ stimulation train at $20 \mathrm{~Hz}$ in $\mathrm{A}_{\beta}^{-1-}$ mice induced a $\Delta F_{\text {peak }}(32 \pm 3 \%, n=5)$ similar to that induced by Train $_{10 \mathrm{~s}}$ in WT, but an $\sim 2$ - to 3 -fold smaller Rate decay $_{\text {. }}$ $(0.37 \pm 0.03 \% / \mathrm{s})$, and a much larger $\Delta F_{100 \mathrm{~s}}(63 \pm 9 \%$, Fig. $7 C)$.

In $\mathrm{A}_{\alpha}^{-1-}$ mice, Train mo s $_{\text {s }}$ induced a $\Delta F_{\text {peak }}$ of $36 \pm 3 \%(n=11)$, a Rate decay $_{\text {of }} 1.02 \pm 0.04 \% / \mathrm{s}(n=11)$ and a $\Delta F_{100 \mathrm{~s}}$ of $2 \pm 1 \%$ $(n=11)$, all of which were similar to the WT (Fig. 7D). We concluded that calcineurin $\mathrm{A}_{\beta}$, but not $\mathrm{A}_{\alpha}$ knock-out inhibits endocytosis in a similar way as CsA at hippocampal synapses (Figs. 6, 7).

Could the lack of effect of $\mathrm{A}_{\alpha}$ knock-out on endocytosis be due to the absence of calcineurin $\mathrm{A}_{\alpha}$ subunit in the hippocampus? To examine this possibility, mouse hippocampal CA1-CA3 regions were dissociated for Western blot using two antibodies against calcineurin $\mathrm{A}_{\alpha}$ and $\mathrm{A}_{\beta}$, respectively (Fig. $7 E$ ). Immunoblotting results revealed that $\mathrm{A}_{\alpha}$ and $\mathrm{A}_{\beta}$ were expressed in wild-type, but not in $\mathrm{A}_{\alpha}^{-1-}$ and $\mathrm{A}_{\beta}^{-1-}$ mice, respectively (Fig. $7 E$ ). Consistent with early studies (Kuno et al., 1992; Hashimoto et al., 1998), these results suggest that the lack of effect of $A_{\alpha}$ knock-out on endocytosis is not due to the absence of $A_{\alpha}$ subunit in the hippocampus.

\section{Discussion}

The present work provided the first genetic evidence together with pharmacological evidence suggesting an important role of calmodulin and calcineurin in rapid and slow endocytosis at 7- to 10 -d-old calyceal synapses and cultured hippocampal synapses (Figs. 1-3, 5-7). Consistent with results obtained at calyces, where calcium influx triggers endocytosis (Hosoi et al., 2009; X. S. Wu et al., $2009)$, reducing the $\left[\mathrm{Ca}^{2+}\right]_{0}$ to $0.1 \mathrm{~mm}$ nearly abolished endocytosis at hippocampal synapses (Fig. 4). We therefore concluded that calcium influx during nerve firings activates calmodulin/calcineurin, 
A

B

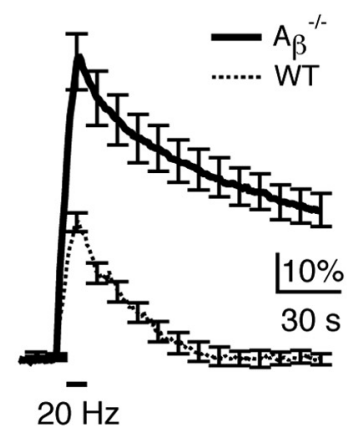

C
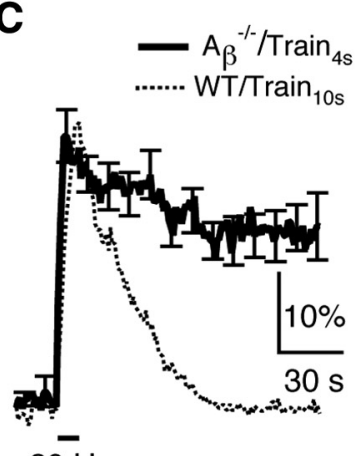

$20 \mathrm{~Hz}$

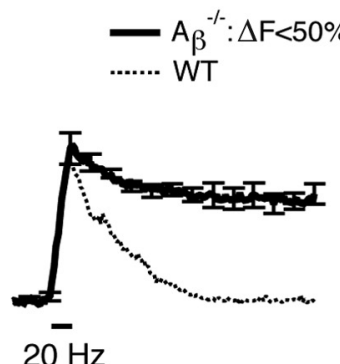

D

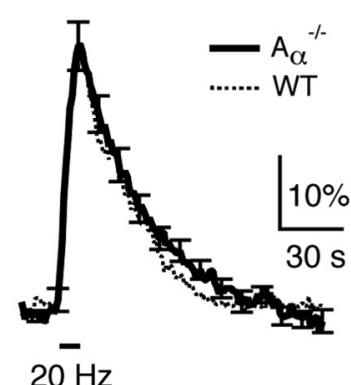

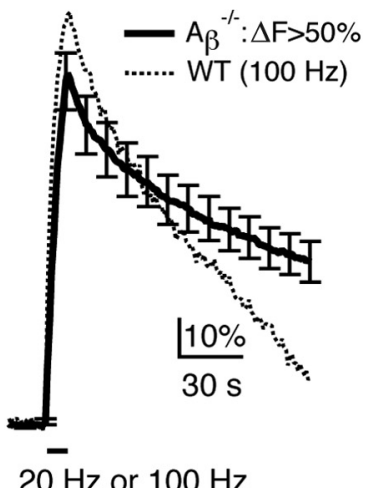

$20 \mathrm{~Hz}$ or $100 \mathrm{~Hz}$

E
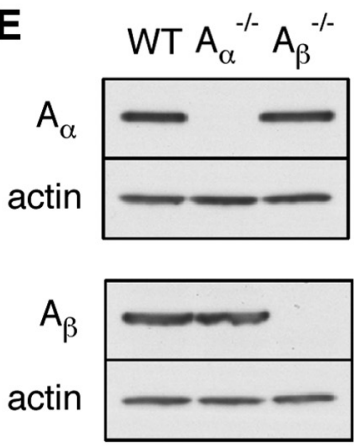

Figure 7. Knock-out of calcineurin $A_{\beta}$ inhibits slow endocytosis at hippocampal synapses. $A$, The SpH signal induced by Train $_{10 \text { s }}$ in calcineurin $\mathrm{A}_{\beta}^{-1-}\left(n=21\right.$, solid) and WT mice $\left(n=4\right.$, dotted). $\boldsymbol{B}$, The experiments in $\mathrm{A}_{\beta}^{-1-}$ mice (thick trace in A) are divided into two groups depending on whether the $\Delta F_{\text {peak }}$ is less than (left, $n=5$ ) or lager than (right, $n=16$ ) $50 \%$ of the baseline (solid). The mean SpH signal induced by Train $_{10}$ in WT is also plotted in the left (dotted), whereas the mean SpH signal induced by a $100 \mathrm{~Hz}$ train for $10 \mathrm{sin}$ WT is plotted in the right (dotted). C, The SpH signal induced by a $20 \mathrm{~Hz}$ train for $4 \mathrm{~s}\left(\operatorname{Train}_{4 \mathrm{~s}}\right)$ in $\mathrm{A}_{\beta}^{-1-}$ mice ( $n=5$, solid). For comparison, the mean SpH signal induced by $\operatorname{Train}_{10 \mathrm{~s}}$ in WT mice (dotted) is also plotted. $\boldsymbol{D}$, The SpH signal induced by Train $_{10 \text { s }}$ in $\mathrm{A}_{\alpha}^{-1-}\left(n=11\right.$, solid) and WT mice $\left(n=4\right.$, dotted). $\boldsymbol{E}$, Western blot of calcineurin $\mathrm{A}_{\alpha}$ (upper) and $\mathrm{A}_{\beta}$ subunit (lower) in the hippocampus (CA3 and CA1 area) of WT, $\mathrm{A}_{\alpha}^{-1-}$ and $\mathrm{A}_{\beta}^{-/-}$mice. Actin is shown as a control.

which initiates and upregulates slow, clathrin-dependent and rapid, presumably clathrin-independent endocytosis.

\section{How does calcineurin control endocytosis?}

Calmodulin/calcineurin-dependent dephosphorylation of endocytic proteins (Robinson et al., 1993; Cousin and Robinson, 2001) may be synchronously activated by calcium influx during nerve firings, which may rapidly increase the endocytosis efficiency and thus initiate endocytosis. Since calcineurin is involved in rapid endocytosis, dephosphorylation must occur within tens to hundreds of milliseconds after stimulation. Larger calcium influx may speed up endocytosis ( $\mathrm{Wu}, 2004)$ by inducing more calmodulin/ calcineurin-dependent dephosphorylation.

How dephosphorylation initiates and accelerates endocytosis is unclear. Dynamin dephosphorylation promotes its interaction with syndapin (Anggono et al., 2006). It was suggested that calcium influx accelerates endocytosis by increasing the number of endocytic sites (Balaji et al., 2008). This suggestion was obtained by reducing the $\left[\mathrm{Ca}^{2+}\right]_{0}$ to only $1 \mathrm{~mm}$. The near full block of endocytosis at $0.1 \mathrm{~mm}\left[\mathrm{Ca}^{2+}\right]_{0}$, as shown here (Fig. 4), suggests an extremely slow endocytosis at each endocytic site, although we could not fully exclude the possibility that few endocytic sites are assembled in low calcium conditions.

Our results seem inconsistent with the observation that endocytosis is triggered by calcium at a threshold $(\sim 10 \mu \mathrm{M})$ higher than the affinity (dissociation constant) of calcineurin to calcium $(\sim 1 \mu \mathrm{M})$ (Rusnak and Mertz, 2000; Hosoi et al., 2009; X. S. Wu et al., 2009). The affinity was measured in vitro with prolonged (minutes) presence of calcium and calcineurin in the steady-state (Rusnak and Mertz, 2000), whereas in nerve terminals, the calcium increase to $>10 \mu \mathrm{M}$ decayed in $<1$ s (Bollmann and Sakmann, 2005; Hosoi et al., 2009). The binding among calcium, calmodulin and calcineurin may not reach the steady-state during transient calcium influx, explaining why $>10 \mu \mathrm{M}$ calcium is needed to initiate endocytosis. Furthermore, calcineurin (B subunit) has four calcium binding sites, one with a high affinity $(<0.1 \mu \mathrm{M})$, and three with affinities at $\sim 15 \mu \mathrm{M}$ (Rusnak and Mertz, 2000), the later of which may help to explain the need of $>10 \mu \mathrm{M}$ calcium.

\section{Calcium/calmodulin/calcineurin controls various forms of endocytosis at many synapses}

Our finding that calcium/calmodulin/calcineurin signaling pathway controls rapid and slow endocytosis may explain regulation of endocytosis by extra- and intracellular calcium observed at many synapses and endocrine cells over the last several decades (Ceccarelli and Hurlbut, 1980; Ramaswami et al., 1994; Henkel and Betz, 1995; Artalejo et al., 1996; Cousin and Robinson, 1998; Gad et al., 1998; Marks and McMahon, 1998; Neves et al., 2001; Sankaranarayanan and Ryan, 2001; W. Wu et al., 2005; Balaji et al., 2008). It may also explain why endocytosis is extremely slow in resting conditions (Hosoi et al., 2009; X. S. Wu et al., 2009). Since calcium/ calmodulin may initiate bulk endocytosis at calyces (X. S. Wu et al., 2009), and calcium/calcineurin may trigger bulk endocytosis at cerebellar synapses (Evans and Cousin, 2007; Clayton and Cousin, 2009; Clayton et al., 2009), it is likely that the calcium/ calmodulin/calcineurin signaling pathway is a common mechanism at synapses to initiate and regulate endocytosis, including rapid, slow, and bulk endocytosis.

Our results seem inconsistent with a report of no calcineurin involvement in slow, clathrin-dependent endocytosis during relatively mild stimulation at cerebellar synapses (Clayton et al., 2009). Although synapse heterogeneity provides an explanation, this discrepancy is likely due to methodological differences. The study at cerebellar synapses was based on the ability of a stimulus to unload FM dye from nerve terminals preloaded with the dye (Clayton et al., 2009). Instead of measuring endocytosis, this method measures the vesicle cycling involving both endocytosis and vesicle reuse. Since the dye was washed out immediately after the dye loading stimulus, the analysis (the amount of dye release after dye preloading) could not provide the endocytosis time course, distinguish between rapid and slow endocytosis, or measure endocytosis time course after stimulation (Clayton et al., 2009). In contrast, we quantitatively measured rapid and slow endocytosis time course using $\mathrm{SpH}$ imaging and capacitance measurement techniques. We used not only calcineurin blockers as in previous studies, but also calcineurin knock-out mice and 
calmodulin knockdown techniques. Furthermore, our results were verified in two types of synapses, the hippocampal and the calyx-type synapse.

Our results seem inconsistent with the block of endocytosis by prolonged intracellular dialysis of $\sim 1 \mu \mathrm{M}$ calcium in ribbon-type synapses (von Gersdorff and Matthews, 1994). Accordingly, our findings are likely limited to the transient calcium increase during brief depolarization. Prolonged calcium increase might perturb the cycle of phosphorylation and dephosphorylation, resulting in a block of endocytosis.

A study published after we finished the present work showed that the calcium buffer BAPTA abolished endocytosis in both the immature (P7-P9) and more mature (P13-P14) calyces (Yamashita et al., 2010), consistent with previous studies (Hosoi et al., 2009; X. S. Wu et al., 2009). This study also showed that calcineurin inhibitors (FK506 and CsA) inhibited rapid and slow endocytosis in P7-P9 calyces (Yamashita et al., 2010), consistent with the present work. Surprisingly, calcineurin inhibitors did not block endocytosis in P13-P14 calyces, suggesting that the calcium sensor for endocytosis changes developmentally from calcineurin to an unknown sensor (Yamashita et al., 2010). Accordingly, our results might be limited to immature synapses. However, this important suggestion may need further scrutiny for two reasons. First, it is based solely on pharmacological manipulation. Second, the same calcium influx triggers both exocytosis and endocytosis (Hosoi et al., 2009; X. S. Wu et al., 2009). Calcium channels are more tightly coupled to release in P13-P14 than P7-P9 calyces, likely because calcium channels are located closer to the release site (Fedchyshyn and Wang, 2005; Wang et al., 2008; Kochubey et al., 2009; Yang et al., 2010). Tight coupling may produce a higher local calcium concentration during the same stimulus, which may accelerate endocytosis to a saturating speed (X. S. Wu et al., 2009). At such high concentration of calcium, the possibility that calcineurin blockers are not as effective in inhibiting endocytosis as in normal conditions has not been ruled out.

\section{Similarity between rapid and slow endocytosis}

Rapid endocytosis is considered clathrin-independent (Artalejo et al., 1995; Jockusch et al., 2005). Its underlying mechanisms are poorly understood. The present work identified calcineurin as an important player in rapid endocytosis. Both rapid and slow endocytosis are regulated by the same calcium/calmodulin/calcineurin signaling pathway (Figs. 1-7) (X. S. Wu et al., 2009), and require dynamin in most, but not some stimulation conditions (Xu et al., 2008). Neither of them recycles vesicles to the readily releasable pool (Wu and $\mathrm{Wu}, 2009)$. These observations suggest that rapid and slow endocytosis share similar mechanisms of initiation, fission, and recycling. Rapid endocytosis is triggered by a higher calcium concentration (Beutner et al., 2001; X. S. Wu et al., 2009), likely because high calcium induces more calcineurindependent dephosphorylation.

\section{Comparison between calyx-type and hippocampal synapses}

Incomplete inhibition of endocytosis by calmodulin and calcineurin blockers, calmodulin knockdown, or calcineurin $\mathrm{A}_{\alpha}$ or $A_{\beta}$ knock-out (Figs. 1-3,5-7) is likely due to the inefficiency of blockers in vivo, the incomplete knockdown of calmodulin, or the remaining calcineurin A subunit. Although the involvement of other calcium-dependent pathway(s) could not be excluded, the calcium/calmodulin/calcineurin pathway must be a major signaling mechanism, because inhibition of calcineurin reduced the Rate $_{\text {decay }}$ by up to $\sim 4$ - to 7 -fold (Figs. 1, 6).
Knock-out of calcineurin $\mathrm{A}_{\alpha}$ and $\mathrm{A}_{\beta}$ inhibited endocytosis at calyces and hippocampal synapses, respectively (Figs. 3, 7). The reason for this difference is unclear. It is not because of the lack of $\mathrm{A}_{\beta}$ at calyces and $\mathrm{A}_{\alpha}$ in the hippocampus, because both isoforms are present in the hippocampus (Fig. 7E) (Kuno et al., 1992; Hashimoto et al., 1998). A difference in the relative abundance or subcellular localization of $\mathrm{A}_{\alpha}$ and $\mathrm{A}_{\beta}$ isoforms might provide an explanation.

CsA and calcineurin $\mathrm{A}_{\beta}$ knock-out increased $\Delta F_{\text {peak }}$ by enhancing transmitter release at hippocampal synapses (Figs. 6, 7), whereas calcineurin inhibitors and calcineurin $\mathrm{A}_{\alpha}$ knock-out did not increase $\Delta C_{\mathrm{m}}$ at calyces (Figs. 1-3). The reason for this difference is unclear. Synapse heterogeneity could provide an explanation. The difference in the stimulation protocol might provide another explanation. If block of calcineurin increases the release probability, but not the readily releasable pool size, it might increase release during action potential stimulation at hippocampal synapses, but not the $\Delta C_{\mathrm{m}}$ induced by $20 \mathrm{~ms}$ depolarization that depleted the readily releasable pool at calyces $(\mathrm{Wu}$ and $\mathrm{Wu}$, 2009).

Knock-out of endocytosis genes often causes behavioral defect. Although we did not examine the behavior of $\mathrm{A}_{\alpha}^{-1-}$ and $\mathrm{A}_{\beta}^{-1-}$ mice, we noticed that we could not generate double knockout mice $\left(\mathrm{A}_{\alpha}^{-1-}, \mathrm{A}_{\beta}^{-1-}\right)$ from $\mathrm{A}_{\alpha}^{-1-}$ and $\mathrm{A}_{\beta}^{-1-}$ mice, likely because they die in the embryonic stage. Consistent with this possibility, knock-out of calcineurin $\mathrm{B}$, the only calcineurin regulatory subunit, results in embryonic death (Chang et al., 2004). Furthermore, most $\mathrm{A}_{\alpha}^{-1-}$ mice die within a few months after birth because of the heart failure (Molkentin et al., 1998). These results suggest the importance of calcineurin for animal survival.

\section{References}

Anggono V, Smillie KJ, Graham ME, Valova VA, Cousin MA, Robinson PJ (2006) Syndapin I is the phosphorylation-regulated dynamin I partner in synaptic vesicle endocytosis. Nat Neurosci 9:752-760.

Artalejo CR, Henley JR, McNiven MA, Palfrey HC (1995) Rapic endocytosis coupled to exocytosis in adrenal chromaffin cells involves $\mathrm{Ca}^{2+}, \mathrm{GTP}$, and dynamin but not clathrin. Proc Natl Acad Sci U S A 92:8328-8332.

Artalejo CR, Elhamdani A, Palfrey HC (1996) Calmodulin is the divalent cation receptor for rapid endocytosis, but not exocytosis, in adrenal chromaffin cells. Neuron 16:195-205.

Atluri PP, Ryan TA (2006) The kinetics of synaptic vesicle reacidification at hippocampal nerve terminals. J Neurosci 26:2313-2320.

Balaji J, Armbruster M, Ryan TA (2008) Calcium control of endocytic capacity at a CNS synapse. J Neurosci 28:6742-6749.

Beutner D, Voets T, Neher E, Moser T (2001) Calcium dependence of exocytosis and endocytosis at the cochlear inner hair cell afferent synapse. Neuron 29:681-690.

Bollmann JH, Sakmann B (2005) Control of synaptic strength and timing by the release-site $\mathrm{Ca}^{2+}$ signal. Nat Neurosci 8:426-434.

Bueno OF, Brandt EB, Rothenberg ME, Molkentin JD (2002) Defective T cell development and function in calcineurin A beta-deficient mice. Proc Natl Acad Sci U S A 99:9398-9403.

Ceccarelli B, Hurlbut WP (1980) $\mathrm{Ca}^{2+}$-dependent recycling of synaptic vesicles at the frog neuromuscular junction. J Cell Biol 87:297-303.

Chang CP, McDill BW, Neilson JR, Joist HE, Epstein JA, Crabtree GR, Chen F (2004) Calcineurin is required in urinary tract mesenchyme for the development of the pyeloureteral peristaltic machinery. J Clin Invest 113:1051-1058.

Chi P, Greengard P, Ryan TA (2003) Synaptic vesicle mobilization is regulated by distinct synapsin I phosphorylation pathways at different frequencies. Neuron 38:69-78.

Clayton EL, Cousin MA (2009) The molecular physiology of activitydependent bulk endocytosis of synaptic vesicles. J Neurochem 111:901-914

Clayton EL, Evans GJ, Cousin MA (2007) Activity-dependent control of bulk endocytosis by protein dephosphorylation in central nerve terminals. J Physiol 585:687-691. 
Clayton EL, Anggono V, Smillie KJ, Chau N, Robinson PJ, Cousin MA (2009) The phospho-dependent dynamin-syndapin interaction triggers activity-dependent bulk endocytosis of synaptic vesicles. J Neurosci 29: 7706-7717.

Cousin MA, Robinson PJ (1998) $\mathrm{Ba}^{2+}$ does not support synaptic vesicle retrieval in rat cerebrocortical synaptosomes. Neurosci Lett 253:1-4.

Cousin MA, Robinson PJ (2001) The dephosphins: dephosphorylation by calcineurin triggers synaptic vesicle endocytosis. Trends Neurosci 24: $659-665$.

Evans GJ, Cousin MA (2007) Activity-dependent control of slow synaptic vesicle endocytosis by cyclin-dependent kinase 5. J Neurosci 27:401-411.

Fedchyshyn MJ, Wang LY (2005) Developmental transformation of the release modality at the calyx of held synapse. J Neurosci 25:4131-4140.

Gad H, Löw P, Zotova E, Brodin L, Shupliakov O (1998) Dissociation between $\mathrm{Ca}^{2+}$-triggered synaptic vesicle exocytosis and clathrin-mediated endocytosis at a central synapse. Neuron 21:607-616.

Gooch JL, Toro JJ, Guler RL, Barnes JL (2004) Calcineurin A-alpha but not A-beta is required for normal kidney development and function. Am J Pathol 165:1755-1765.

Granseth B, Odermatt B, Royle SJ, Lagnado L (2006) Clathrin-mediated endocytosis is the dominant mechanism of vesicle retrieval at hippocampal synapses. Neuron 51:773-786.

Hashimoto T, Kawamata T, Saito N, Sasaki M, Nakai M, Niu S, Taniguchi T, Terashima A, Yasuda M, Maeda K, Tanaka C (1998) Isoform-specific redistribution of calcineurin $\mathrm{A}$ alpha and $\mathrm{A}$ beta in the hippocampal CA1 region of gerbils after transient ischemia. J Neurochem 70:1289-1298.

Henkel AW, Betz WJ (1995) Monitoring of black widow spider venom (BWSV) induced exo- and endocytosis in living frog motor nerve terminals with FM1-43. Neuropharmacology 34:1397-1406.

Hosoi N, Holt M, Sakaba T (2009) Calcium dependence of exo- and endocytotic coupling at a glutamatergic synapse. Neuron 63:216-229.

Jockusch WJ, Praefcke GJ, McMahon HT, Lagnado L (2005) Clathrindependent and clathrin-independent retrieval of synaptic vesicles in retinal bipolar cells. Neuron 46:869-878.

Kochubey O, Han Y, Schneggenburger R (2009) Developmental regulation of the intracellular $\mathrm{Ca} 2+$ sensitivity of vesicle fusion and $\mathrm{Ca} 2+$-secretion coupling at the rat calyx of Held. J Physiol 587:3009-3023.

Kuno T, Mukai H, Ito A, Chang CD, Kishima K, Saito N, Tanaka C (1992) Distinct cellular expression of calcineurin A alpha and A beta in rat brain. J Neurochem 58:1643-1651.

Lin MJ, Lin-Shiau SY (1999) Enhanced spontaneous transmitter release at murine motor nerve terminals with cyclosporine. Neuropharmacology 38:195-198.

Marks B, McMahon HT (1998) Calcium triggers calcineurin-dependent synaptic vesicle recycling in mammalian nerve terminals. Curr Biol 8:740-749.

Molkentin JD, Lu JR, Antos CL, Markham B, Richardson J, Robbins J, Grant SR, Olson EN (1998) A calcineurin-dependent transcriptional pathway for cardiac hypertrophy. Cell 93:215-228.

Neves G, Gomis A, Lagnado L (2001) Calcium influx selects the fast mode of endocytosis in the synaptic terminal of retinal bipolar cells. Proc Natl Acad Sci U S A 98:15282-15287.

Newton AJ, Kirchhausen T, Murthy VN (2006) Inhibition of dynamin completely blocks compensatory synaptic vesicle endocytosis. Proc Natl Acad Sci U S A 103:17955-17960.

Oliveria SF, Dell'Acqua ML, Sather WA (2007) AKAP79/150 anchoring of calcineurin controls neuronal L-type $\mathrm{Ca}^{2+}$ channel activity and nuclear signaling. Neuron 55:261-275.

Pang ZP, Cao P, Xu W, Südhof TC (2010) Calmodulin controls synaptic strength via presynaptic activation of CaM kinase II. J Neurosci 30: $4132-4142$
Ramaswami M, Krishnan KS, Kelly RB (1994) Intermediates in synaptic vesicle recycling revealed by optical imaging of Drosophila neuromuscular junctions. Neuron 13:363-375.

Robinson PJ, Sontag JM, Liu JP, Fykse EM, Slaughter C, McMahon H, Südhof TC (1993) Dynamin GTPase regulated by protein kinase C phosphorylation in nerve terminals. Nature 365:163-166.

Royle SJ, Lagnado L (2003) Endocytosis at the synaptic terminal. J Physiol 553:345-355.

Rusnak F, Mertz P (2000) Calcineurin: form and function. Physiol Rev 80: 1483-1521.

Sakaba T, Neher E (2001) Calmodulin mediates rapid recruitment of fastreleasing synaptic vesicles at a calyx-type synapse. Neuron 32:1119-1131.

Sankaranarayanan S, Ryan TA (2000) Real-time measurements of vesicleSNARE recycling in synapses of the central nervous system. Nat Cell Biol 2:197-204.

Sankaranarayanan S, Ryan TA (2001) Calcium accelerates endocytosis of vSNAREs at hippocampal synapses. Nat Neurosci 4:129-136.

Sihra TS, Nairn AC, Kloppenburg P, Lin Z, Pouzat C (1995) A role for calcineurin (protein phosphatase-2B) in the regulation of glutamate release. Biochem Biophys Res Commun 212:609-616.

Sun JY, Wu LG (2001) Fast kinetics of exocytosis revealed by simultaneous measurements of presynaptic capacitance and postsynatpic currents at a central synapse. Neuron 30:171-182.

Sun JY, Wu XS, Wu LG (2002) Single and multiple vesicle fusion induce different rates of endocytosis at a central synapse. Nature 417:555-559.

Sun JY, Wu XS, Wu W, Jin SX, Dondzillo A, Wu LG (2004) Capacitance measurements at the calyx of Held in the medial nucleus of the trapezoid body. J Neurosci Methods 134:121-131.

von Gersdorff H, Matthews G (1994) Inhibition of endocytosis by elevated internal calcium in a synaptic terminal. Nature 370:652-655.

Wang LY, Neher E, Taschenberger H (2008) Synaptic vesicles in mature calyx of Held synapses sense higher nanodomain calcium concentrations during action potential-evoked glutamate release. J Neurosci 28:14450-14458.

Wu LG (2004) Kinetic regulation of vesicle endocytosis at synapses. Trends Neurosci 27:548-554.

Wu LG, Betz WJ (1996) Nerve activity but not intracellular calcium determines the time course of endocytosis at the frog neuromuscular junction. Neuron 17:769-779.

Wu LG, Ryan TA, Lagnado L (2007) Modes of vesicle retrieval at ribbon synapses, calyx-type synapses, and small central synapses. J Neurosci 27:11793-11802.

Wu W, Xu J, Wu XS, Wu LG (2005) Activity-dependent acceleration of endocytosis at a central synapse. J Neurosci 25:11676-11683.

Wu XS, Wu LG (2009) Rapid endocytosis does not recycle vesicles within the readily releasable pool. J Neurosci 29:11038-11042.

Wu XS, McNeil BD, Xu J, Fan J, Xue L, Melicoff E, Adachi R, Bai L, Wu LG (2009) $\mathrm{Ca}(2+)$ and calmodulin initiate all forms of endocytosis during depolarization at a nerve terminal. Nat Neurosci 12:1003-1010.

Xu J, McNeil B, Wu W, Nees D, Bai L, Wu LG (2008) GTP-independent rapid and slow endocytosis at a central synapse. Nat Neurosci 11:45-53.

Yamashita T, Eguchi K, Saitoh N, von Gersdorff H, Takahashi T (2010) Developmental shift to a mechanism of synaptic vesicle endocytosis requiring nanodomain Ca2 +. Nat Neurosci 13:838-844.

Yang YM, Fedchyshyn MJ, Grande G, Aitoubah J, Tsang CW, Xie H, Ackerley CA, Trimble WS, Wang LY (2010) Septins regulate developmental switching from microdomain to nanodomain coupling of $\mathrm{Ca}(2+)$ influx to neurotransmitter release at a central synapse. Neuron 67:100-115.

Zhang BW, Zimmer G, Chen J, Ladd D, Li E, Alt FW, Wiederrecht G, Cryan J, O'Neill EA, Seidman CE, Abbas AK, Seidman JG (1996) T cell responses in calcineurin A alpha-deficient mice. J Exp Med 183:413-420. 